Gabriela Guevara-Carrion, Robin Fingerhut, Jadran Vrabec

\title{
Fick Diffusion Coefficient Matrix of a Quaternary Liquid Mixture by Molecular Dynamics
}

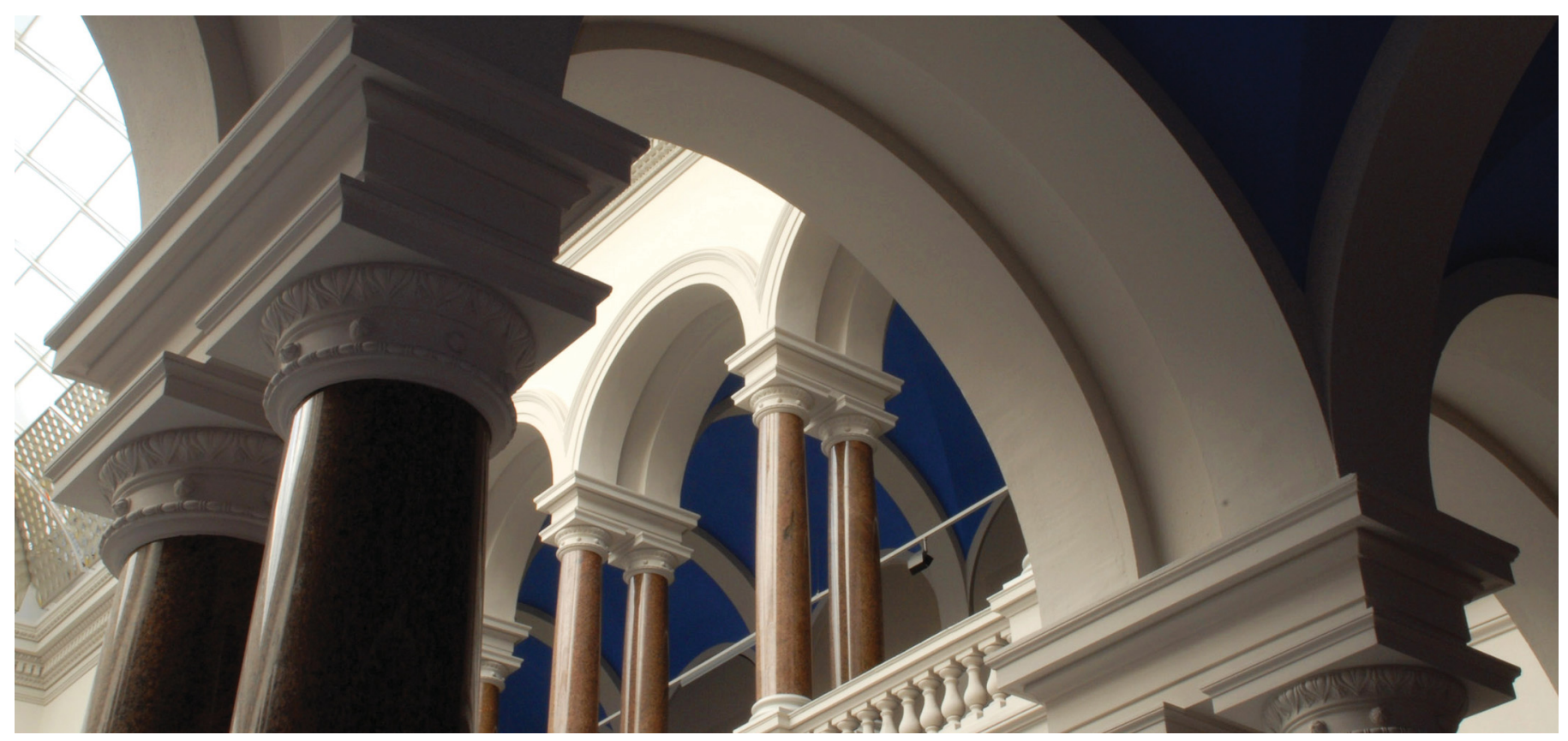

Guevara-Carrion, G., Fingerhut, R., \& Vrabec, J. (2020). Fick Diffusion Coefficient Matrix of a Quaternary Liquid Mixture by Molecular Dynamics. The Journal of Physical Chemistry B, 124(22), 4527-4535. https://doi.org/10.1021/acs.jpcb.0c01625 


\section{Fick Diffusion Coefficient Matrix of a}

\section{Quaternary Liquid Mixture by Molecular Dynamics}

Gabriela Guevara-Carrion, Robin Fingerhut, and Jadran Vrabec*

Thermodynamics and Process Engineering, Technical University Berlin, 10587 Berlin, Germany

E-mail: vrabec@tu-berlin.de

Phone: +493031422755 


\begin{abstract}
For the first time, the Fick diffusion coefficient matrix of a quaternary liquid mixture is sampled consistently by means of molecular dynamics simulation. The required phenomenological diffusion coefficient and thermodynamic factor matrices of the mixture water + methanol + ethanol + 2-propanol are determined following the Green-Kubo formalism and KirkwoodBuff theory. Further, a system size correction methodology for the Fick diffusion coefficient of multicomponent mixtures is proposed. Ten compositions are studied under ambient conditions and validated by analyzing the ternary limits of the quaternary Fick diffusion matrix. Because of complex intermolecular interactions due to the presence of hydrogen bonding, the elements of the Fick diffusion coefficient matrix exhibit a significant composition dependence. The magnitude of several cross coefficients indicate important coupling effects mainly affecting the diffusive flux of water. These effects are explained in the light of the structural information given by the radial distribution functions of the mixture. This work that solely rests on molecular dynamics simulation techniques to predict the Fick diffusion coefficient matrix of quaternary mixtures is expected to be a significant step forward for the understanding of multicomponent diffusion.
\end{abstract}

\title{
Graphical TOC Entry
}

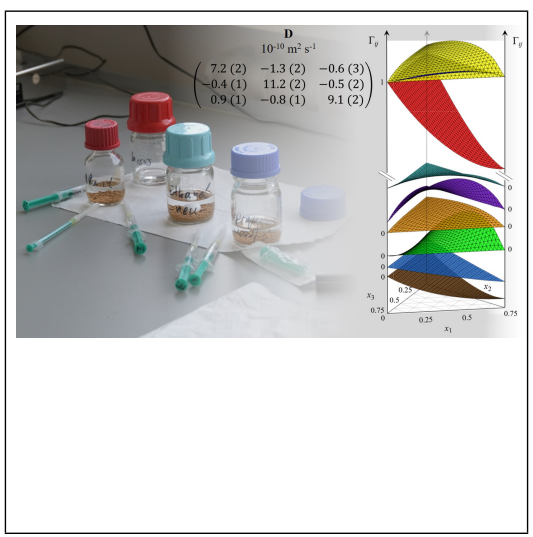




\section{Introduction}

Multicomponent solutions are involved in the majority of mass transfer processes occurring in nature and technical applications ${ }^{1}$. Almost all separation processes in chemical engineering, such as distillation, absorption or extraction, are affected by multicomponent diffusion in liquids. Ratebased methods employed for modeling, design and control of these unit operations involve mass and energy transfer models, which require diffusion coefficient data for mixtures ${ }^{2}$.

Mass transport in multicomponent systems is complex. For instance, describing isothermalisobaric diffusion of a quaternary mixture by Fick's law requires a matrix with nine different diffusion coefficient elements that are composition dependent. Related experimental work is challenging not only because of the elaborate equipment that is required to distinguish between different components, but also because of the presence of coupling effects that hinder data processing and interpretation $^{3}$. Consequently, the availability of experimental data on diffusion coefficients for mixtures containing three or more components is very limited ${ }^{4}$. In fact, they have experimentally been measured only for 17 quaternary mixtures ${ }^{5-17}$, but in some of these cases, only the main elements of the diffusion matrix are reported. With diffusion having entered the scientific arena in the 1850s through the contributions of Graham ${ }^{18}$ and Fick ${ }^{19}$, experimental measurements alone are obviously not able to satisfy the growing need for accurate mass transport properties ${ }^{20}$, particularly for liquids that are constituted of many components.

Most predictive equations for diffusion coefficients of multicomponent liquids are extensions of the Darken relation ${ }^{21-23}$, which is not valid for mixtures with strong intermolecular interactions. The underlying physical phenomena are not well understood and the lack of experimental data impedes the development and verification of new predictive equations. On the other hand, molecular dynamics simulation is a compelling alternative for such predictions. In fact, such simulations have the capability to accurately predict Fick diffusion coefficients of binary ${ }^{24,25}$ and ternary mixtures $^{26,27}$. Krishna and van Baten ${ }^{21}$ were the first to deduce the required mathematical framework and sampled the Maxwell-Stefan diffusion coefficient matrix of quaternary n-alkane mixtures with molecular dynamics. Later, Liu et al. ${ }^{22}$ determined the Maxwell-Stefan diffusion coefficients of 
quaternary model mixtures interacting with the Weeks-Chandler-Andersen potential. However, none of these works predicted the Fick diffusion coefficient, perhaps because of the lack of a mathematical framework to sample the required thermodynamic factor matrix. In a recent work of our group ${ }^{28}$, expressions for the thermodynamic factor matrix of quaternary mixtures based on Kirkwood-Buff theory were derived to close this gap. To the best of our knowledge, this is the first work on the prediction of quaternary Fick diffusion coefficients solely based on molecular dynamics simulation techniques.

The quaternary mixture water + methanol + ethanol + 2-propanol was chosen in this context since the Fick diffusion coefficient of most of the involved subsystems has been predicted in previous work. In fact, the binary subsystems water + methanol $^{29}$, water + ethanol $^{29}$, methanol + ethanol ${ }^{30}$, water +2 -propanol ${ }^{25}$, and the ternary subsystem water + methanol + ethanol $^{26,27}$ have successfully been studied in this sense.

\section{Theory}

In the framework of the generalized form of Fick's law, the molar flux of component $i$ in a mixture of four components is written as a linear combination of concentration gradients $\nabla c_{j}{ }^{31}$

$$
J_{i}=-\sum_{j=1}^{3} D_{i j} \nabla c_{j}, \quad(i=1,2,3),
$$

where $D_{i i}$ are the main diffusion coefficients that relate the molar flux of component $i$ to its own concentration gradient and $D_{i j}$ are the cross diffusion coefficients that relate the molar flux of component $i$ to the concentration gradient of component $j^{32}$. The Fick approach involves three independent diffusion fluxes and a $3 \times 3$ diffusion coefficient matrix, which is generally not symmetric, i.e. $D_{i j} \neq D_{j i}$. Further, the numerical values of $D_{i j}$ depend both on the reference frame for velocity (molar-, mass- or volume-averaged) and on the order of the components. In this work, the molar-averaged reference frame is employed throughout.

The main shortcoming of Fick's law is the fact that concentration gradients are not the true 
thermodynamic driving forces for diffusion, which are rather given by chemical potential gradients. Maxwell-Stefan theory follows this path, assuming that chemical potential gradients $\nabla \mu_{i}$ are balanced by friction forces between the components that are proportional to their mutual velocity $\left(\boldsymbol{u}_{i}-\boldsymbol{u}_{j}\right)^{33}$

$$
\sum_{j \neq i=1}^{4} \frac{x_{j}\left(\boldsymbol{u}_{i}-\boldsymbol{u}_{j}\right)}{\bigoplus_{i j}}=-\frac{1}{k_{\mathrm{B}} T} \nabla \mu_{i} \quad(i, j=1, \ldots, 4),
$$

where $x_{j}$ is the mole fraction of component $j, k_{\mathrm{B}}$ Boltzmann's constant and $T$ the temperature. The Maxwell-Stefan diffusion coefficient $\bigoplus_{i j}$ plays the role of an inverse friction coefficient between components $i$ and $j^{34}$. Its matrix does not depend on the component order and is symmetric so that it has only six independent elements.

Maxwell-Stefan diffusion coefficients are associated with chemical potential gradients and thus cannot directly be measured in the laboratory. However, they are accessible with equilibrium molecular simulation techniques, i.e. the Green-Kubo formalism ${ }^{35,36}$ or the Einstein approach ${ }^{37}$. For multicomponent mixtures, Fick and Maxwell-Stefan diffusion coefficients are related by ${ }^{33}$

$$
\mathbf{D}=\mathbf{B}^{-1} \cdot \boldsymbol{\Gamma}
$$

in which all three symbols represent $3 \times 3$ matrices and the elements of $\mathbf{B}$ are given by ${ }^{21}$

$$
B_{i i}=\frac{x_{i}}{\bigoplus_{i 4}}+\sum_{j \neq i=1}^{4} \frac{x_{j}}{\bigoplus_{i j}}, \quad B_{i j}=-x_{i}\left(\frac{1}{\bigoplus_{i j}}-\frac{1}{\bigoplus_{i 4}}\right) .
$$

Fick diffusion coefficients can be calculated from the Maxwell-Stefan diffusion coefficients if the thermodynamic factor matrix $\Gamma$

$$
\Gamma_{i j}=\delta_{i j}+\left.x_{i} \frac{\partial \ln \gamma_{i}}{\partial x_{j}}\right|_{T, p, x_{k, k \neq j=1 \ldots 3}}
$$

is known. Therein, $\delta_{i j}$ is the Kronecker delta function and $\gamma_{i}$ the activity coefficient of component $i$. The partial derivative has to be evaluated at constant temperature, pressure and mole fraction of 
all other components.

\section{Ternary limits of quaternary Fick diffusion coefficients}

The consistency of the present simulation results can be assessed by analyzing the asymptotic behavior of quaternary diffusion coefficients when approaching the ternary limits. This type of analysis has been developed for ternary mixtures ${ }^{27,38}$ and is expanded here to quaternary mixtures. Following the previously outlined procedure ${ }^{27}$, the asymptotic behavior of some elements of the quaternary Fick diffusion coefficient matrix in the molar-averaged reference frame can be obtained. When the water content of the studied quaternary mixture water (1) + methanol (2) + ethanol (3) +2 -propanol (4) vanishes, $\nabla x_{1} \rightarrow 0$, the mixture approaches its ternary subsystem methanol + ethanol + 2-propanol. By comparing the diffusive flux equations of the quaternary and ternary mixtures, it follows that

$$
D_{22}^{\text {quat }} \rightarrow D_{11}^{\text {tern }}, \quad D_{23}^{\text {quat }} \rightarrow D_{12}^{\text {tern }}, \quad D_{32}^{\text {quat }} \rightarrow D_{21}^{\text {tern }}, \quad D_{33}^{\text {quat }} \rightarrow D_{22}^{\text {tern }},
$$

where $D_{i j}^{\text {quat }}$ and $D_{i j}^{\text {tern }}$ denote the respective elements of the quaternary and ternary Fick diffusion coefficient matrices.

From a similar analysis for vanishing methanol content, $x_{2} \rightarrow 0$, it follows for the ternary limit water + ethanol +2 -propanol that

$$
D_{11}^{\text {quat }} \rightarrow D_{11}^{\text {tern }}, \quad D_{13}^{\text {quat }} \rightarrow D_{12}^{\text {tern }}, \quad D_{31}^{\text {quat }} \rightarrow D_{21}^{\text {tern }}, \quad D_{33}^{\text {quat }} \rightarrow D_{22}^{\text {tern }} .
$$

Analogously, when ethanol disappears from the mixture, $x_{3} \rightarrow 0$, the asymptotic behavior towards the ternary limit water + methanol +2 -propanol is given by 


$$
D_{21}^{\text {quat }} \rightarrow D_{21}^{\text {tern }}, \quad D_{22}^{\text {quat }} \rightarrow D_{22}^{\text {tern }}, \quad D_{11}^{\text {quat }} \rightarrow D_{11}^{\text {tern }}, \quad D_{12}^{\text {quat }} \rightarrow D_{12}^{\text {tern }} .
$$

The behavior of quaternary diffusion when approaching the ternary subsystem water + methanol + ethanol, $x_{4} \rightarrow 0$, requires some transformation of the expressions for the diffusive fluxes. Employing $x_{1}+x_{2}+x_{3}+x_{4}=1$ and $\nabla x_{3}=-\nabla x_{1}-\nabla x_{2}-\nabla x_{4}$, it follows that

$$
\begin{array}{ll}
\left(D_{11}^{\text {quat }}-D_{13}^{\text {quat }}\right) \rightarrow D_{11}^{\text {tern }}, & \left(D_{12}^{\text {quat }}-D_{13}^{\text {quat }}\right) \rightarrow D_{12}^{\text {tern }}, \\
\left(D_{21}^{\text {quat }}-D_{23}^{\text {quat }}\right) \rightarrow D_{21}^{\text {tern }}, & \left(D_{22}^{\text {quat }}-D_{23}^{\text {quat }}\right) \rightarrow D_{22}^{\text {tern }} .
\end{array}
$$

A detailed derivation of Eqs. (6) to (9) is given in the supplementary material.

\section{Methods}

The Fick diffusion coefficient matrix of quaternary and ternary mixtures was calculated from Eqs. (3) to (5) and matrices $\mathbf{B}$ and $\Gamma$ that were sampled exclusively by means of equilibrium molecular dynamics simulation techniques.

The primary requirement for this task is the availability of molecular models that mimic the intermolecular interactions adequately. In this work, rigid and non-polarizable force fields of unitedatom type were employed, which account for these interactions by a set of Lennard-Jones sites and point charges which may or may not coincide with respect to their site positions. The molecular models for the three alcohols were developed by our group based on quantum chemical calculations and parameter optimization to experimental vapor-liquid equilibrium and, in the case of 2-propanol, also to self-diffusion data $25,39-41$. For water, the TIP4P/2005 model by Abascal and Vega $^{39}$ was employed. This force field was found to predict the transport properties of water and aqueous alcoholic mixtures with a better accuracy than other commonly used non-polarizable force 
fields ${ }^{29}$. The interested reader is referred to the original publications ${ }^{25,39-41}$ for detailed information about the four molecular pure substance models and their parameters. It has been shown that all molecular models are suitable for the prediction of structural, thermodynamic and transport properties of the corresponding pure substances ${ }^{25,29,30}$ as well as four of the binary $25,26,30$ and one of the ternary ${ }^{26,27}$ subsystems of the regarded quaternary mixture.

To define a molecular model for a mixture on the basis of pairwise additive pure substance models, only the unlike interactions have to be specified. In case of the point charges, this can straightforwardly be done with Coulomb's law. However, for the unlike Lennard-Jones parameters, there is no physically sound approach so that combining rules have to be employed. The simple Lorentz-Berthelot combining rules were chosen here, i.e., $\sigma_{a b}=\left(\sigma_{a a}+\sigma_{b b}\right) / 2$ and $\varepsilon_{a b}=\sqrt{\varepsilon_{a a} \varepsilon_{b b}}$, so that the present mixture data are strictly predictive.

\section{Phenomenological coefficients}

Transport data were sampled by equilibrium molecular dynamics simulation and the Green-Kubo formalism ${ }^{35,36}$ based on the net velocity auto-correlation function to obtain the $4 \times 4$ phenomenological coefficient matrix 21

$$
L_{i j}=\frac{1}{3 N} \int_{0}^{\infty} \mathrm{dt}\left\langle\sum_{k=1}^{N_{i}} \mathbf{v}_{i, k}(0) \cdot \sum_{l=1}^{N_{j}} \mathbf{v}_{j, l}(\mathrm{t})\right\rangle .
$$

Here, $N$ is the total number of molecules, $N_{i}$ the number of molecules of component $i$ and $\mathbf{v}_{i, k}(t)$ the center of mass velocity vector of the $k$-th molecule of component $i$ at time $t$. The brackets $<\ldots>$ denote the canonical (NVT) ensemble average and Eq. (10) corresponds to a reference frame in which the mass-averaged velocity of the mixture is zero ${ }^{21}$.

With the phenomenological coefficients $L_{i j}$, the elements of a $3 \times 3$ matrix $\Delta$ can be defined ${ }^{21}$

$$
\Delta_{i j}=\left(1-x_{i}\right)\left(\frac{L_{i j}}{x_{j}}-\frac{L_{i 4}}{x_{4}}\right)-x_{i} \sum_{k=1 \neq i}^{4}\left(\frac{L_{k j}}{x_{j}}-\frac{L_{k 4}}{x_{4}}\right),
$$

This matrix can be directly employed in Eq. (3), since it is related to the matrix B by its inverse, 
$\mathbf{B}=\boldsymbol{\Delta}^{-1}$.

\section{System size corrections}

It has been shown that under periodic boundary conditions, long-range interactions can lead to an important dependence on system size inducing systematic errors on the calculation of the selfdiffusion or intra-diffusion coefficients ${ }^{42-45}$. Yeh and Hummer ${ }^{43}$ found that hydrodynamic selfinteractions in finite periodic systems are mainly responsible for system size effects and derived a correction term based on the shear viscosity $\eta$ and the edge length of the simulation volume $L$, i.e. $2.837297 \cdot k_{B} T /(6 \pi \eta L)$. Later on, Heyes et al. ${ }^{44}$ performed an investigation of hard sphere fluids over a wide range of thermodynamic conditions and concluded that the Yeh and Hummer correction term is not always adequate and that a more complex density dependence is needed. Recently, Jamali et al. ${ }^{46}$ proposed a correction for the Maxwell-Stefan diffusion coefficient based

on the Yeh and Hummer ${ }^{43}$ term and the thermodynamic factor, but this correction is only valid for binary mixtures. To the best of our knowledge, there has been no attempt to perform any system size corrections for mutual diffusion coefficients of multicomponent mixtures.

Instead of applying the system size corrections directly to the Maxwell-Stefan or Fick diffusion coefficients, they were rather applied here to the phenomenological coefficients $L_{i j}$, cf. Eq. (11). Once their system size effects were assessed, corrected values of the phenomenological coefficients were employed in Eq. (3) to obtain the corresponding Fick diffusion coefficients. For this purpose, simulations were performed for nine system sizes containing between 512 and 6000 molecules for compositions of the present mixture with the lowest and highest molar density. The normalized simulation results were then plotted over $1 / N^{1 / 3}$ to asses the system size dependence. For all phenomenological cross coefficients $L_{i j}$, no clear system size dependence could be inferred, which might be due to their large statistical uncertainty, so that no corrections were made. In the case of the four main coefficients $L_{i i}$, a significant system size dependence was observed. A straight line fitted to these data was extrapolated to infinite size, $1 / N^{1 / 3} \rightarrow 0$. Very similar size corrections for all $L_{i i}$ coefficients were found. Consequently, all main coefficients were normalized with the 
results for 6000 molecules and plotted together in Figure 1. The resulting intercept of a single linear fit was then employed to obtain the relative size correction for all sampled main coefficients $L_{i i}$. Note that no density effects were observed. The size correction calculated in this way led to an increase of the phenomenological coefficients $L_{i i}$ by approximately $6 \%$. A correction following Yeh and Hummer would have led to around $15 \%$ larger corrections.

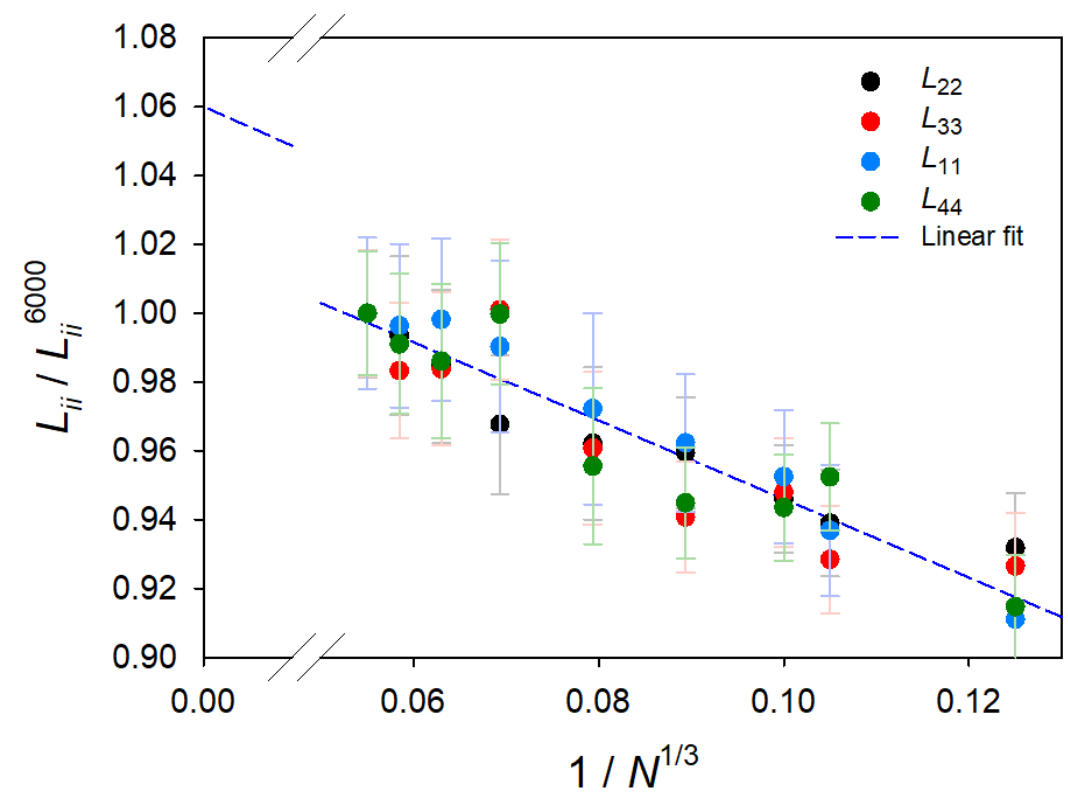

Figure 1: System size dependence of the main phenomenological coefficients $L_{i i}$ of the quaternary mixture $x_{1}=0.5 \mathrm{~mol} \mathrm{~mol}^{-1}, x_{2}=0.125 \mathrm{~mol} \mathrm{~mol}^{-1}$ and $x_{3}=0.125 \mathrm{~mol} \mathrm{~mol}^{-1}$ at $298.15 \mathrm{~K}$ and 0.1 MPa.

\section{Thermodynamic factor}

The thermodynamic factor of the quaternary mixture was estimated from information on the microscopic structure given by radial distribution functions $g_{i j}(r)$ based on Kirkwood-Buff theory. Kirkwood-Buff integrals $G_{i j}$ are defined in the grand canonical $(\mu V T)$ ensemble $^{47}$ by

$$
G_{i j}=4 \pi \int_{0}^{\infty}\left(g_{i j}(r)-1\right) r^{2} d r
$$


When the canonical (NVT) ensemble is employed to sample this type of property, convergence issues are possible ${ }^{48}$ so that corrections are required. In this context, the truncation and correction method developed by Krüger et al. ${ }^{49}$, that allows to obtain Kirkwood-Buff integrals from simulations in the $N V T$ ensemble, was applied here. Moreover, corrections of the radial distribution functions are required. Therefore, Kirkwood-Buff integrals were calculated based on the methodology proposed by Ganguly and van der Vegt ${ }^{50}$, which was found to be the most adequate in previous work ${ }^{51}$. Extrapolation to the thermodynamic limit was not necessary because of the rather large ensemble size $N=6000$.

Expressions for the thermodynamic factor matrix of quaternary mixtures were derived in recent work $^{28}$ based on Ben-Naim's formalism to determine particle number derivatives of the chemical potential from Kirkwood-Buff integrals ${ }^{52}$. These lengthly equations have recently been published elsewhere ${ }^{28}$ are not repeated here.

\section{Results and Discussion}

Predictive equilibrium molecular dynamics simulations of diffusion coefficients and the thermodynamic factor of the quaternary mixture water (1) + methanol (2) + ethanol (3) + 2-propanol (4) and its pure, binary and ternary and subsystems were carried out at $298.15 \mathrm{~K}$ and $0.1 \mathrm{MPa}$ for the compositions depicted in Figure 2. A total of ten quaternary compositions of this mixture, that lay on the mole fraction plane $x_{4}=0.25 \mathrm{~mol} \mathrm{~mol}^{-1}$, are discussed in this work.

To validate the simulation results from Kirkwood-Buff theory for the quaternary mixture, the thermodynamic factor of all six binary subsystems was analyzed. For this purpose, additional simulations were performed to sample the thermodynamic factor for all involved binary subsystems and compared with the values obtained from a classical approach, i.e. a fit of the Wilson excess Gibbs energy model ${ }^{53}$ to experimental vapor-liquid equilibrium data. A good agreement was found between simulation and the classical approach, suggesting the trustworthiness of the employed simulation methodology to access the chemical potential derivatives of the quaternary 


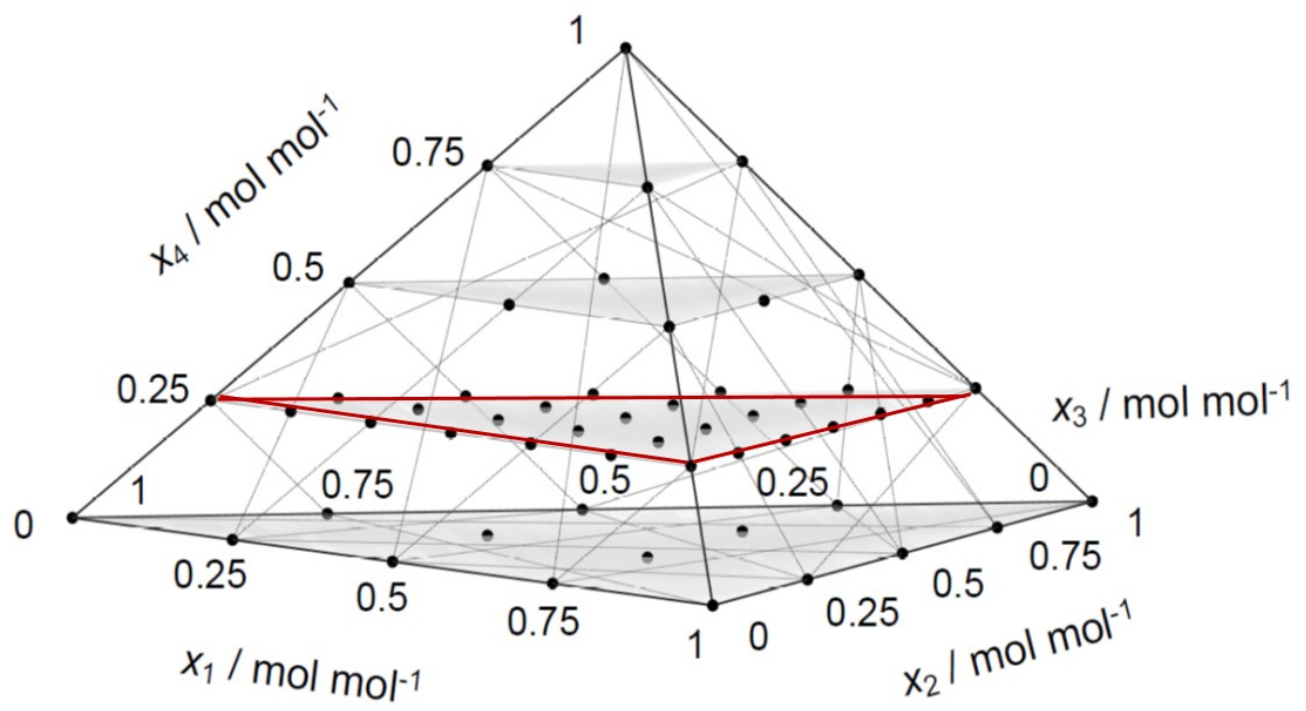

Figure 2: Sampled compositions of the quaternary mixture water (1) + methanol (2) + ethanol (3) + 2-propanol (4) at 298.15 K and 0.1 MPa. The surface delimited by red lines indicates the plane with a constant 2-propanol mole fraction $x_{4}=0.25 \mathrm{~mol} \mathrm{~mol}^{-1}$. 
mixture. Moreover, the Wilson model ${ }^{53}$ was fitted to data of the binary subsystems and the obtained binary parameters were employed to predict the quaternary thermodynamic factor matrix for the studied compositions. The resulting Wilson-based quaternary thermodynamic factor matrix is shown in comparison with the one sampled directly with molecular dynamics for selected compositions in Figure 3. More comparisons of this type are provided in the supplementary material. A good agreement was found especially for the main elements of this matrix with a relative averages deviations between 2.3 and $3.6 \%$.

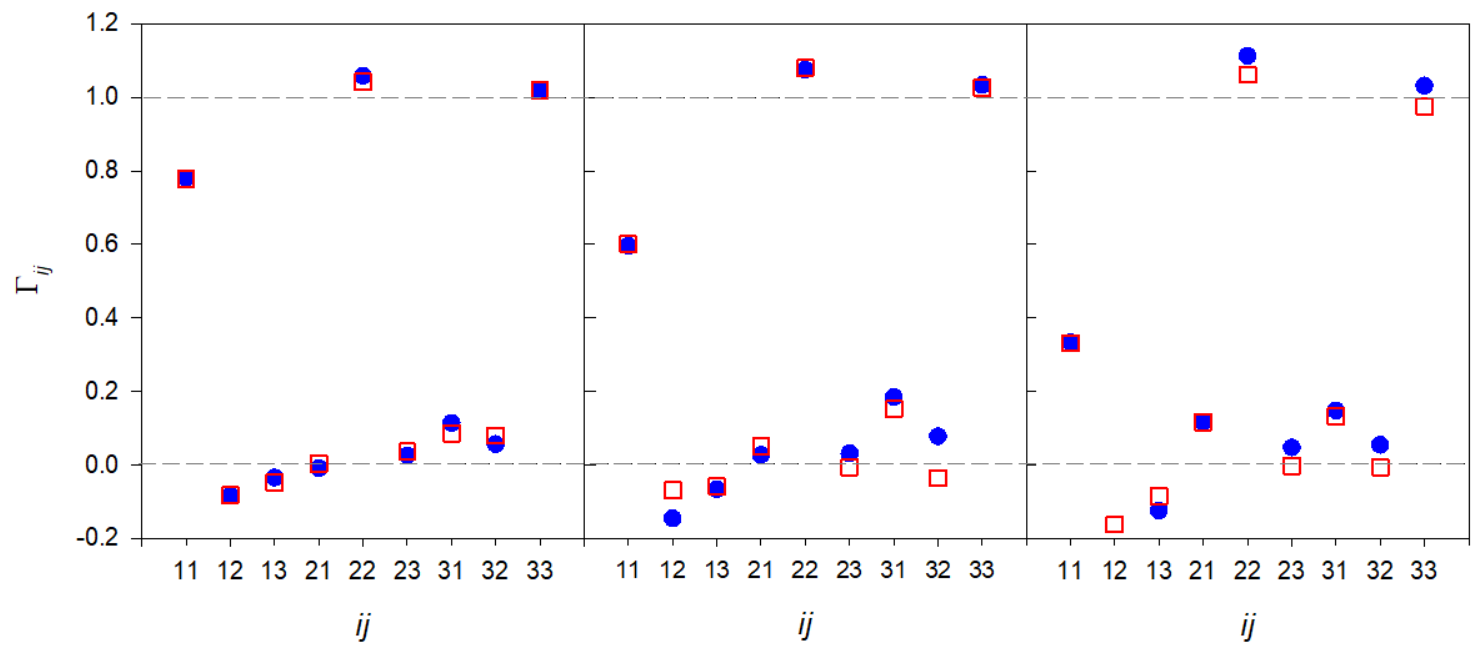

Figure 3: Elements of the thermodynamic factor matrix $\Gamma_{i j}$ for three selected quaternary compositions; left: $x_{1}=0.125 \mathrm{~mol} \mathrm{~mol}^{-1}, x_{2}=0.125 \mathrm{~mol} \mathrm{~mol}^{-1}$ and $x_{3}=0.5 \mathrm{~mol} \mathrm{~mol}^{-1}$; center: $x_{1}=$ $0.25 \mathrm{~mol} \mathrm{~mol}^{-1}, x_{2}=0.125 \mathrm{~mol} \mathrm{~mol}^{-1}$ and $x_{3}=0.375 \mathrm{~mol} \mathrm{~mol}^{-1}$; right: $x_{1}=0.5 \mathrm{~mol} \mathrm{~mol}^{-1}, x_{2}=$ $0.125 \mathrm{~mol} \mathrm{~mol}^{-1}$ and $x_{3}=0.125 \mathrm{~mol} \mathrm{~mol}^{-1}$. Present quaternary results based on Kirkwood-Buff integration (red symbols) are compared with $\Gamma_{i j}$ calculated with the Wilson excess Gibbs energy model fitted to binary simulation results (blue symbols). The statistical uncertainties are within symbol size.

The main element $\Gamma_{11}$ of the thermodynamic factor matrix related to water evidences the highest non-ideality, yielding values that are considerably lower than unity, while the other two main elements attain values close to unity. The cross elements of the thermodynamic factor matrix can be of either sign and have values ranging from -0.2 to 0.2 for the studied compositions, cf. Figure 3 .

The Fick diffusion matrix calculated with Eq. (3) for ten compositions of the quaternary mix- 
ture is given in Table 1. Its main elements are positive and have values between 3.7 and $12.7 \times 10^{-10}$ $\mathrm{m}^{2} \mathrm{~s}^{-1}$. Further, the main element related to methanol is the highest and that related to water the lowest, i.e. $D_{22}>D_{33}>D_{11}$. Lower values for the main diffusion coefficients of water can be associated with the presence of clustering, exerted by strong hydrogen bonding networks, which hinders diffusion.

In order to visualize and test the consistency of the calculated Fick quaternary diffusion coefficient matrix, selected elements were plotted when the mole fraction of two species is kept constant in the mixture. Exemplarly, Figure 4 shows the composition dependence of main and cross elements together with their expected asymptotic values, cf. Eqs. (6) to (8). Additional figures are presented in the supplementary material. In most cases, the elements of the Fick diffusion coefficient matrix behave as expected, i.e. the sampled quaternary data converge to the projected value when one species vanishes. This trend is clear for the main coefficients, but the cross coefficients scatter, which may be a consequence to their inherently larger statistical uncertainties.

The main diffusion coefficient related to water $D_{11}$ increases with increasing methanol content. This can be explained by the disturbance of the microscopic water-water structure through the presence of water-methanol hydrogen bonding and is evidenced by changes in the magnitude and location of the first and second peaks of the center of mass water-water radial distribution function $g_{11}(r)$, cf. Figure 5. A similar observation was made for the somewhat weaker increase of $D_{11}$ with rising ethanol content; the corresponding radial distribution functions are shown in the supplementary material.

The decrease of $D_{22}$ and $D_{33}$ with rising water content is consistent with the presence of watermethanol and water-ethanol networks, which can be also deduced from the corresponding radial distribution functions $g_{12}(r)$ and $g_{13}(r)$. When the water content is kept constant, the main coefficient related to ethanol $D_{33}$ decreases with rising ethanol mole fraction due to a strengthening of the ethanol-ethanol hydrogen bonding network, cf. Figure 4. A small decrease of $D_{22}$ with rising ethanol mole fraction was also observed for a constant water content, which suggests an increase of the presence of methanol-ethanol networks when the number methanol molecules available for 
Table 1: Fick diffusion coefficient matrix and its three eigenvalues of the quaternary mixture water (1) + methanol (2) + ethanol (3) + 2-propanol (4) at 298.15 K and 0.1 MPa. The numbers in parentheses indicate the uncertainty in the last given digit.

\begin{tabular}{|c|c|c|c|c|c|c|}
\hline$x_{1}$ & $\begin{array}{c}x_{2} \\
\text { ol } \mathrm{mol}^{-}\end{array}$ & $x_{3}^{x_{3}}$ & & $\begin{array}{c}\text { D } \\
10^{-10} \mathrm{~m}^{2} \mathrm{~s}^{-}\end{array}$ & & $\begin{array}{c}\hat{\mathbf{D}} \\
10^{-10} \mathrm{~m}^{2} \mathrm{~s}^{-1}\end{array}$ \\
\hline 0.125 & 0.125 & 0.5 & $\left(\begin{array}{l}7.1(2) \\
0.0(2) \\
0.2(2)\end{array}\right.$ & $\begin{array}{r}-1.0(3) \\
10.9(3) \\
-0.7(4)\end{array}$ & $\left.\begin{array}{r}-0.7(1) \\
0.3(1) \\
8.9(2)\end{array}\right)$ & $\left.\begin{array}{r}7.1 \\
10.8 \\
8.9\end{array}\right)$ \\
\hline 0.125 & 0.25 & 0.375 & $\left(\begin{array}{r}7.9(2) \\
0.4(2) \\
-0.4(2)\end{array}\right.$ & $\begin{array}{r}-1.0(2) \\
10.8(2) \\
-0.3(3)\end{array}$ & $\begin{array}{r}-0.4(2) \\
-0.2(2) \\
9.2(2)\end{array}$ & $\left(\begin{array}{r}7.8 \\
10.7 \\
9.3\end{array}\right)$ \\
\hline 0.125 & 0.375 & 0.25 & $\left(\begin{array}{l}8.6(2) \\
0.1(3) \\
0.0(2)\end{array}\right.$ & $\begin{array}{r}-1.2(2) \\
12.7(2) \\
-0.7(2)\end{array}$ & $\left.\begin{array}{r}-0.7(2) \\
-0.1(2) \\
11.0(2)\end{array}\right)$ & $\begin{array}{r}8.6 \\
12.7 \\
11.0\end{array}$ \\
\hline 0.125 & 0.5 & 0.125 & $\left(\begin{array}{r}9.8(2) \\
0.0(2) \\
-0.3(2)\end{array}\right.$ & $\begin{array}{r}-0.9(2) \\
12.6(2) \\
-0.6(1)\end{array}$ & $\begin{array}{r}-0.7(2) \\
-0.6(3) \\
11.3(2)\end{array}$ & $\begin{array}{r}9.6 \\
12.8 \\
11.3\end{array}$ \\
\hline 0.25 & 0.125 & 0.375 & $\left(\begin{array}{r}5.8(1) \\
1.3(1) \\
-0.5(1)\end{array}\right.$ & $\begin{array}{r}-0.9(3) \\
10.3(2) \\
-1.5(3)\end{array}$ & $\begin{array}{r}-0.5(2) \\
-0.1(1) \\
7.9(1)\end{array}$ & $\left.\begin{array}{r}6.1 \\
10.2 \\
7.7\end{array}\right)$ \\
\hline 0.25 & 0.25 & 0.25 & $\left(\begin{array}{l}6.3(1) \\
0.7(1) \\
0.0(1)\end{array}\right.$ & $\begin{array}{c}-1.5(2) \\
10.4(2) \\
-0.6(2)\end{array}$ & $\begin{array}{r}-0.6(2) \\
-0.1(2) \\
8.5(2)\end{array}$ & $\left.\begin{array}{r}6.6 \\
10.1 \\
8.4\end{array}\right)$ \\
\hline 0.25 & 0.375 & 0.125 & $\left(\begin{array}{r}7.2(1) \\
-0.4(1) \\
0.9(1)\end{array}\right.$ & $\begin{array}{r}-1.3(2) \\
11.2(2) \\
-0.8(1)\end{array}$ & $\begin{array}{r}-0.6(3) \\
-0.5(3) \\
9.1(2)\end{array}$ & $\left(\begin{array}{r}7.4 \\
11.5 \\
8.6\end{array}\right)$ \\
\hline 0.375 & 0.125 & 0.25 & $\begin{array}{c}4.3(1) \\
1.3(1) \\
-0.28(8)\end{array}$ & $\begin{array}{r}-1.6(3) \\
9.0(2) \\
-0.7(2)\end{array}$ & $\begin{array}{r}-1.1(2) \\
-0.1(1) \\
7.4(2)\end{array}$ & $\left(\begin{array}{c}4.8 \\
8.7 \\
7.2\end{array}\right)$ \\
\hline 0.375 & 0.25 & 0.125 & $\begin{array}{l}5.1(1) \\
0.1(1) \\
0.97(7)\end{array}$ & $\begin{array}{r}-2.0(3) \\
10.2(2) \\
-0.4(1)\end{array}$ & $\begin{array}{r}-1.1(3) \\
0.1(2) \\
8.0(2)\end{array}$ & $\left(\begin{array}{r}5.5 \\
10.1 \\
7.6\end{array}\right)$ \\
\hline 0.5 & 0.125 & 0.125 & $\begin{array}{l}3.7(1) \\
0.55(6) \\
0.41(5)\end{array}$ & $\begin{array}{r}-2.3(3) \\
8.6(2) \\
-0.3(1)\end{array}$ & $\begin{array}{r}-0.8(3) \\
-0.3(2) \\
6.6(1)\end{array}$ & $\left(\begin{array}{l}4.2 \\
8.5 \\
6.2\end{array}\right)$ \\
\hline
\end{tabular}



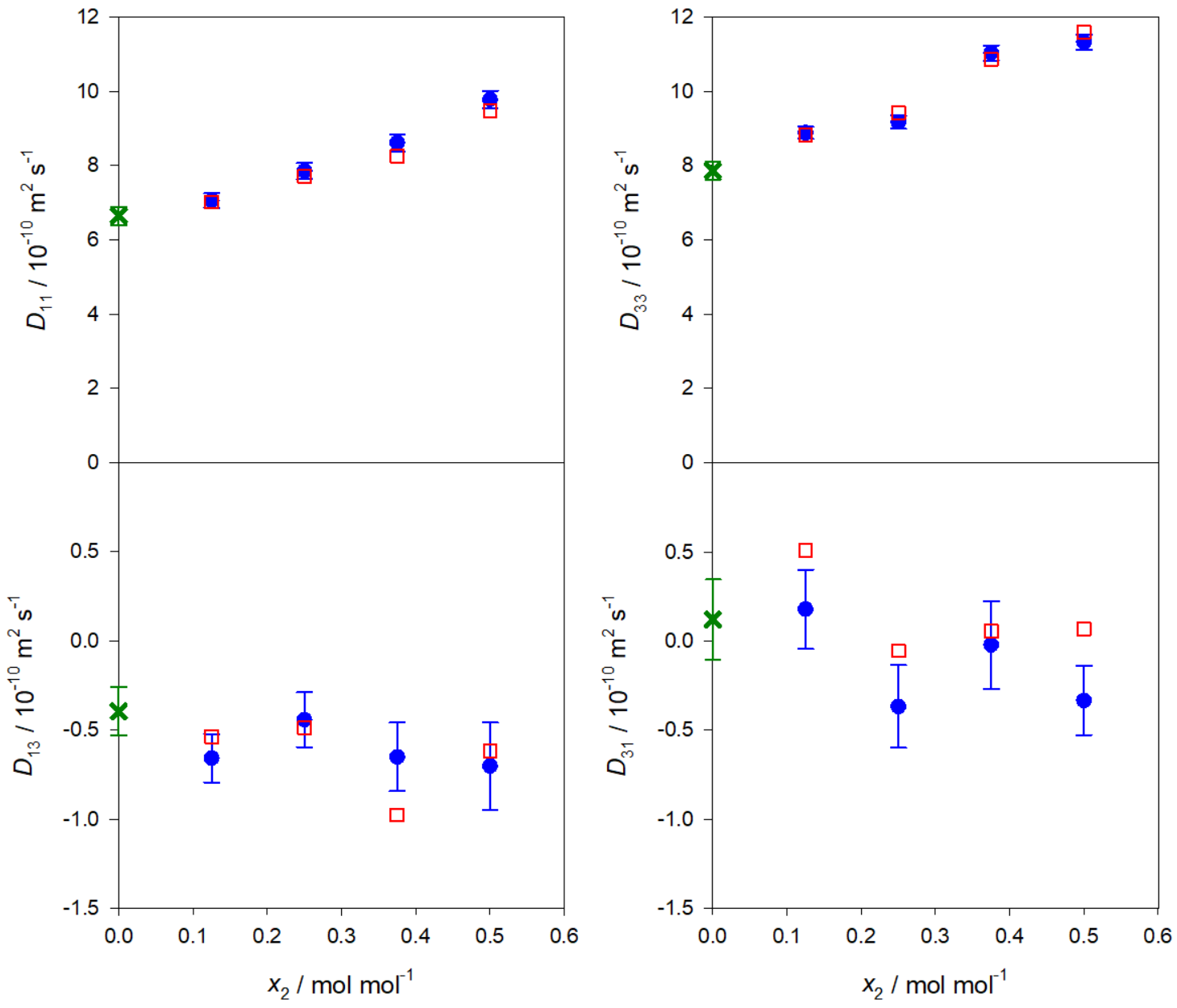

Figure 4: Main and cross elements of the Fick diffusion coefficient matrix as a function of methanol mole fraction for selected compositions with $x_{1}=0.125 \mathrm{~mol} \mathrm{~mol}^{-1}$ and $x_{4}=0.25 \mathrm{~mol} \mathrm{~mol}^{-1}$. Simulation results for the quaternary mixture (blue symbols) are shown together with the results of the diffusion matrix of the ternary subsystem where $x_{2} \rightarrow 0$ (green symbol) and the predictive equations by Allie-Ebrahim et al. ${ }^{23}$ (red symbols). 


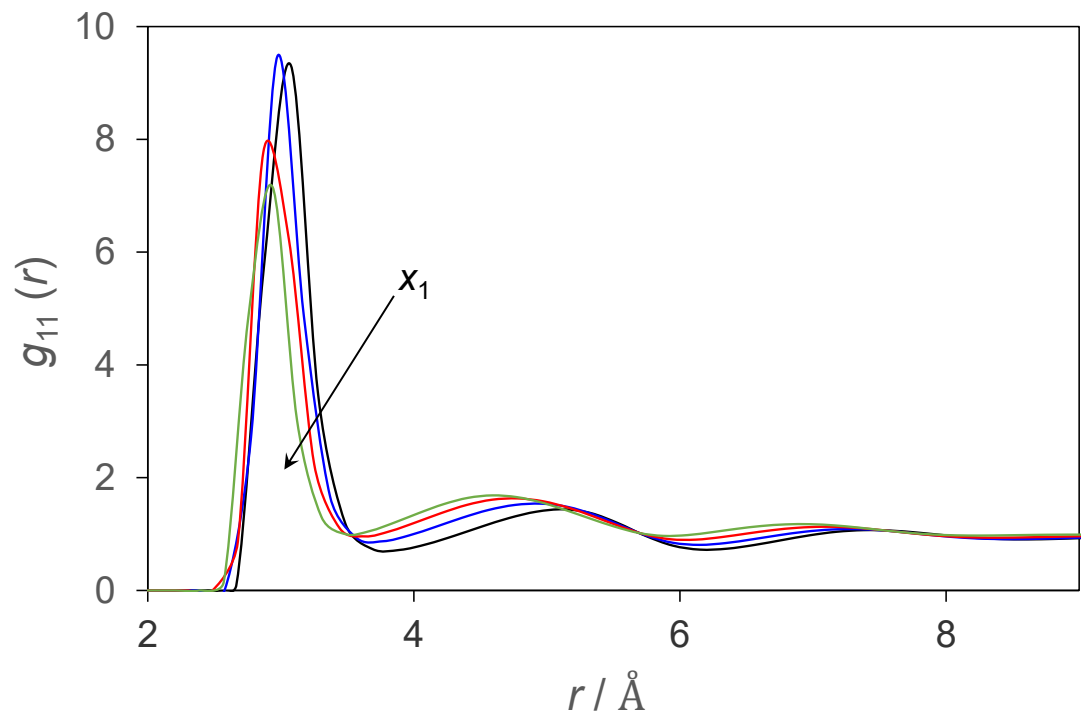

Figure 5: Water-water radial distribution function $g_{11}(r)$ for varying water mole fraction $x_{1}=0.125$ mol mol ${ }^{-1}$ (black line), $0.25 \mathrm{~mol} \mathrm{~mol}^{-1}$ (blue line), $0.375 \mathrm{~mol} \mathrm{~mol}^{-1}$ (red line) and $0.5 \mathrm{~mol} \mathrm{~mol}^{-1}$ (green line) with constant $x_{3}=0.125 \mathrm{~mol} \mathrm{~mol}^{-1}$ and $x_{4}=0.25 \mathrm{~mol} \mathrm{~mol}^{-1}$. 
self-association is reduced. This explanation is in line with the changes observed for the radial distribution function $g_{23}(r)$, i.e. an increase of the radii of the first and second coordination shells, cf. supplementary material.

For most of the studied compositions, the cross elements $D_{i j}$ are not zero, i.e. there is an influence of the concentration gradient of another species on the diffusion of component $i$. In fact, both cross coefficients related to water have the same order of magnitude as the main coefficient $D_{11}$, which might lead to important coupling effects when a significant concentration gradient of the corresponding alcohol is present. The ratio between cross and main elements of the Fick diffusion coefficient matrix $\left|D_{i j} / D_{i i}\right|$ provides a characterization of coupling effects. Figure 6 shows the composition dependence of this ratio for the water-related cross coefficients when the mole fraction of two alcohols remains constant. In general, $\left|D_{12} / D_{11}\right|$ increases with water content, which suggests important water clustering around methanol by hydrogen bonding. In the case of the cross coefficient $D_{13}$, the tendency is not that clear, although a moderate increase with water mole fraction can be inferred. Further, the influence of the concentration gradient of methanol on water diffusion is greater than that of ethanol. This observation could be explained by the larger hydrophobic tail of ethanol molecules, which reduce the occurrence and size of mixed waterethanol clusters.

In order to demonstrate the relationship between the microscopic structure and the coupling effects, the average number of water(1) molecules in the first solvation shell of both alcohols $k_{i 1}=$ $4 \pi x_{i} \rho \int_{0}^{r_{c}} g_{i j}(r) r^{2} d r$ was calculated. Therein, $i$ stands for the methanol or ethanol site surrounded by water, $\rho$ is the mixture density and $r_{c}$ is the radius of the first coordination shell, i.e. the location of the first minimum of $g_{i 1}$ at $r_{c} \sim 3.6 \AA$. These results are shown together with those from the ratio $\left|D_{i j} / D_{i i}\right|$ in Figure 6. It is clear that the coupling effects are related to the average number of water molecules located in the first solvation shell of methanol or ethanol, i.e. water molecules are transported together with methanol or ethanol molecules when a mass flux of either alcohol is given.

On the other hand, most cross coefficients related to $D_{22}$ and $D_{33}$ are relatively small and the 


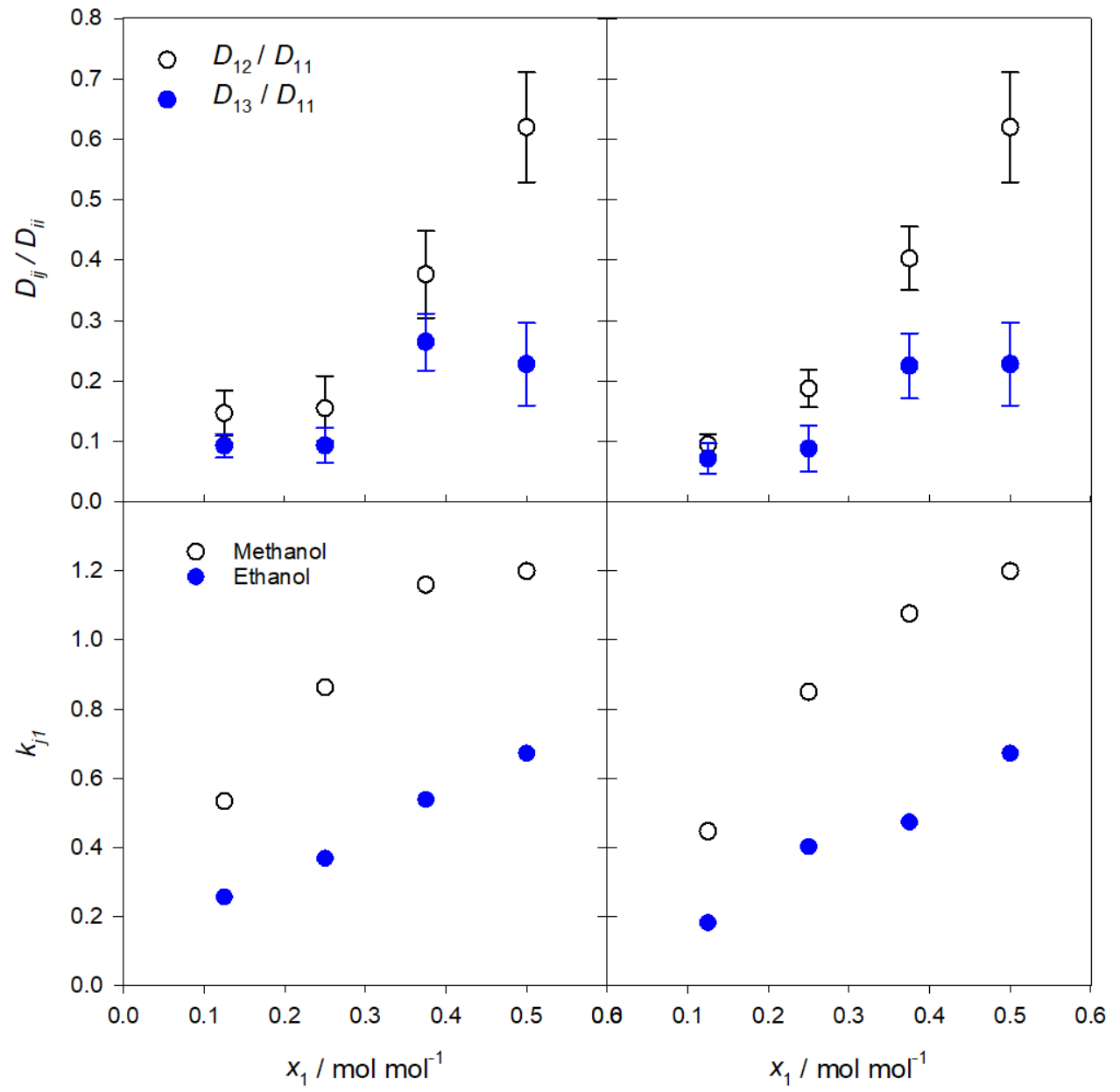

Figure 6: Ratio between cross and main elements of the Fick diffusion coefficient matrix $\left|D_{i j} / D_{i i}\right|$ (top) and average number of water molecules in the first solvation shell of methanol or ethanol (bottom) as a function of water mole fraction for selected quaternary compositions with $x_{2}=0.125$ mol mol ${ }^{-1}$ and $x_{4}=0.25 \mathrm{~mol} \mathrm{~mol}^{-1}$ (left) as well as $x_{3}=0.125 \mathrm{~mol} \mathrm{~mol}^{-1}$ and $x_{4}=0.25 \mathrm{~mol}$ $\mathrm{mol}^{-1}$ (right). 
Fick matrix eigenvalues approach the values of the main coefficients, nearly implying an independence of the diffusion fluxes of methanol and ethanol on the concentration gradients of the other species, cf. Table 1.

The intra-diffusion coefficients of the four species in the mixture were sampled simultaneously with the phenomenological diffusion coefficients. These data, together with the thermodynamic factor, were employed to test the Darken-based predictive equations by Liu et al. ${ }^{22}$ and AllieEbrahim et al. ${ }^{23}$ on their ability to reproduce the Fick diffusion coefficients calculated in this work. While the predictive equation by Liu et al. ${ }^{22}$ was not able to return acceptable results, the equations of Allie-Ebrahim et al. ${ }^{23}$ are in very good agreement with the present results for the main elements of the Fick diffusion matrix with relative average deviations below 10\%, cf. Figure 4. Further, in most cases there is also an agreement for the cross elements within the statistical uncertainties of the simulation data.

\section{Conclusions}

Diffusion processes in multicomponent liquid mixtures are of great importance in science and engineering research. However, because of the serious difficulties associated with the measurement of transport diffusion coefficients, the data availability for mixtures with three or more components is very poor. Molecular dynamics simulation has been identified as an alternative to mitigate data shortage for ternary mixtures. For the first time, as a powerful approach to Fick diffusion coefficients of real quaternary liquid mixtures is presented here.

The Fick diffusion coefficient matrix of the strongly non-ideal liquid mixture water + methanol + ethanol + 2-propanol at 298.15 K and 0.1 MPa was sampled for ten compositions along the plane with constant 2-propanol mole fraction, $x_{4}=0.25 \mathrm{~mol} \mathrm{~mol}^{-1}$. The required phenomenological coefficient matrix was calculated with the Green-Kubo formalism ${ }^{35,36}$ and the thermodynamic factor matrix employing Kirkwood-Buff theory. Because of the lack of experimental data, only consistency tests were made to verify the sampled Fick diffusion coefficients. However, present 
predictions are expected to be accurate because convincing results were obtained in previous work for four binary and one of the ternary subsystems on the basis of the same molecular models. The asymptotic behavior of the elements of the quaternary Fick diffusion coefficient matrix was analyzed and found to agree within the statistical uncertainties with the values of the ternary subsystems.

From the analysis of the Fick diffusion matrix, significant coupling effects were found mainly for the diffusive flux for water, which were related to water-alcohol hydrogen bonding networks. The lowest values for the main coefficient of water and its decrease with rising water content were explained with the presence of water clusters.

Two Darken-based predictive models were tested and the predictive equations of Allie-Ebrahim et al. ${ }^{23}$ were found to be in very good agreement with the Fick diffusion matrix predicted in this work.

\section{Acknowledgement}

This work contributes to the Collaborative Research Center SFB-TRR 75 of Deutsche Forschungsgemeinschaft (DFG) and was funded under Grant No. VR 6/11. We gratefully acknowledge the

Paderborn Center for Parallel Computing $\left(\mathrm{PC}^{2}\right)$ for the generous allocation of computer time on the OCuLUS and Noctua clusters as well as the High Performance Computing Center Stuttgart (HLRS) under the grant MMHBF2.

\section{Supporting Information Available}

Contains the detailed calculation of the ternary limits of quaternary Fick diffusion coefficients as well the simulation procedure. The complete set of Figures for the main and cross elements of the Fick diffusion coefficient matrix for the quaternary mixture as shown in Figure 4. The graphical representation of selected radial distribution functions. 


\section{References}

(1) Miller, D. G. Some Comments on Multicomponent Diffusion: Negative Main Term Diffusion Coefficients, Second Law Constraints, Solvent Choices, and Reference Frame Transformations. J. Phys. Chem. 1986, 90, 1509-1519.

(2) Kenig, E. Y.; Blagov, S. In Distillation: Fundamentals and Principles; Gòrak, A., Sorensen, E., Eds.; Elsevier: London, 2014.

(3) Guevara-Carrion, G.; Ancherbak, S.; Mialdun, A.; Vrabec, J.; Shetsova, V. Diffusion of Methane in Supercritical Carbon Dioxide Across the Widom Line. Sci. Rep. 2019, 9, 8466.

(4) Wambui Mutoru, J.; Firoozabadi, A. Form of Multicomponent Fickian Diffusion Coefficients Matrix. J. Chem. Thermodyn. 2011, 43, 1192-1203.

(5) Rai, G. P.; Cullinan, H. T. Diffusion Coefficients of Quaternary Liquid System AcetoneBenzene-Carbon Tetrachloride-n-Hexane at $25{ }^{0}$ C. J. Chem. Eng. Data 1973, 18, 213-214.

(6) Noulty, R. A.; Leaist, D. G. Quaternary Diffusion in Aqueous Potassium Chloride-Potassium Dihydrogen Phosphate-Phosphoric Acid Mixtures. J. Phys. Chem. 1987, 91, 1655-1658.

(7) Leaist, D. G. Diaphragm-Cell Studies of Diffusion in the Four-Component System Hydrochloric Acid-Sodium Chloride-Sodium Iodide-Water. J. Chem. Soc. Faraday Trans. 1 1987, 83, 829-839.

(8) Leaist, D. G. Diffusion Coefficients of Four-Component Systems from Taylor Dispersion Profiles. Sucrose $+\mathrm{LiCl}+\mathrm{KCl}+$ Water and $\mathrm{NBu} 4 \mathrm{Br}+\mathrm{LiBr}+\mathrm{KBr}+$ Water. Ber. Bunsenges. Phys. Chem. 1991, 95, 117-122.

(9) Paduano, L.; Sartorio, R.; Vitagliano, V.; Albright, J. G.; Miller, D. G. Measurement of the Mutual Diffusion Coefficients at One Composition of the Four-Component System $\alpha$-Cyclodextrln-L-Rhenylalanlne-Monobutylurea- $\mathrm{H}_{2} \mathrm{O}$ at $25{ }^{0} \mathrm{C}$. J. Phys. Chem. 1992, 96, $7478-7483$. 
(10) Leaist, D. G.; Hao, L. Quaternary Diffusion Coefficients of $\mathrm{NaCl}-\mathrm{MgCl}_{2}-\mathrm{Na}_{2} \mathrm{SO}_{4}-\mathrm{H}_{2} \mathrm{O}$ Synthetic Seawaters by Least-Squares Analysis of Taylor Dispersion Profiles. J. Solution Chem. 1993, 22, 263-277.

(11) McEwan, K.; Leaist, D. G. Quaternary Mutual Diffusion Coefficients for Aqueous Solutions of a Cationic-Anionic Mixed Surfactant from Moments Analysis of Taylor Dispersion Profiles. Phys. Chem. Chem. Phys. 2003, 18, 3951-3958.

(12) Annunziata, O.; Vergara, A.; Paduano, L.; Sartorio, R.; Miller, D. G.; Albright, J. G. Precision of Interferometric Diffusion Coefficients in a Four Component System Relevant to Protein Crystal Growth: Lysozyme-Tetra(ethylene glycol)-NaCl- $\mathrm{H}_{2} \mathrm{O}$. J. Phys. Chem. B 2003, 107, 6590-6597.

(13) Vanag, V. K.; Rossi, F.; Cherkashin, A.; Epstein, I. R. Cross-Diffusion in a Water-in-Oil Microemulsion Loaded with Malonic Acid or Ferroin. Taylor Dispersion Method for FourComponent Systems. J. Phys. Chem. B 2008, 112, 9058-9070.

(14) Annunziata, O.; Vergara, A.; Paduano, L.; Sartorio, R.; Miller, D. G.; Albright, J. G. Quaternary Diffusion Coefficients in a Protein-Polymer-Salt-WaterSystem Determined by Rayleigh Interferometry. J. Phys. Chem. B 2009, 113, 13446-13456.

(15) Ribeiro, A. C. F.; Santos, C. I. A. V.; Lobo, V. M. M.; Esteso, M. A. Quaternary Diffusion Coefficients of $\beta$-Cyclodextrin $+\mathrm{KCl}+$ Caffeine + Water at 298.15 K Using a Taylor Dispersion Method. J. Chem. Eng. Data 2010, 55, 2610-2612.

(16) Rossi, F.; Vanag, V. K.; Tiezzi, E.; Epstein, I. R. Quaternary Cross-Diffusion in Water-in-Oil Microemulsions Loaded with a Component of the Belousov-Zhabotinsky Reaction. J. Phys. Chem. B 2010, 114, 8140-8146.

(17) Peters, C.; Thien, J.; Wolff, L.; Koß, H.-J.; Bardow, A. Quaternary Diffusion Coefficients in Liquids from Microfluidics and Raman Microspectroscopy: Cyclohexane + Toluene + Acetone + Methanol. J. Chem. Eng. Data 2020, 65, 1273-1288. 
(18) Graham, T. The Bakerian Lecture.- On the Diffusion of Liquids. Philos. Trans. R. Soc. 1850, $140,1-46$.

(19) Fick, A. E. Über Diffusion. Pogg. Ann. Phys. Chem. 1855, 94, 59-86.

(20) Wakeham, W. A. In Chemical Thermodynamics for Industry; Letcher, T. M., Ed.; The Royal Society of Chemistry: London, 2004.

(21) Krishna, R.; van Baten, J. M. The Darken Relation for Multicomponent Diffusion in Liquid Mixtures of Linear Alkanes: An Investigation Using Molecular Dynamics (MD) Simulations. Ind. Eng. Chem. Res. 2005, 44, 6939-6847.

(22) Liu, X.; Vlugt, T. J. H.; Bardow, A. Predictive Darken Equation for Maxwell-Stefan Diffusivities in Multicomponent Mixtures. Ind. Eng. Chem. Res. 2011, 50, 10350-10358.

(23) Allie-Ebrahim, T.; Russo, V.; Ortona, O.; Paduano, L.; Tesser, R.; Di Serio, M.; Singh, P.; Zhu, Q.; Moggridge, G. D.; D’Agostino, C. A Predictive Model for the Diffusion of a Highly Non-Ideal Ternary System. Phys. Chem. Chem. Phys. 2018, 20, 18436-18446.

(24) Guevara-Carrion, G.; Janzen, T.; Munoz-Munoz, Y. M.; Vrabec, J. Mutual diffusion of Binary Liquid Mixtures Containing Methanol, Ethanol, Acetone, Benzene, Cyclohexane, Toluene, and Carbon Tetrachloride. J. Chem. Phys. 2016, 144, 124501.

(25) Muñoz-Muñoz, Y. M.; Guevara-Carrion, G.; Vrabec, J. Molecular Insight into the Liquid Propan-2-ol +Water Mixture. J. Phys. Chem. B 2018, 122, 8718-8729.

(26) Parez, S.; Guevara-Carrion, G.; Hasse, H.; Vrabec, J. Mutual Diffusion in the Ternary Mixture of Water + Methanol + Ethanol and its Binary Subsystems. Phys. Chem. Chem. Phys. 2013, $15,3985-4001$.

(27) Guevara-Carrion, G.; Gaponenko, Y. A.; Janzen, T.; Vrabec, J.; Shetsova, V. Diffusion in Multicomponent Liquids: From Microscopic to Macroscopic Scales. J. Phys. Chem. B 2016, $120,12193-12210$. 
(28) Fingerhut, R.; Herres, G.; Vrabec, J. Thermodynamic Factor of Quaternary Mixtures from Kirkwood-Buff Integration. Mol. Phys. 2020, doi: 10.1080/00268976.2019.1643046.

(29) Guevara-Carrion, G.; Vrabec, J.; Hasse, H. Prediction of Self-diffusion Coefficient and Shear Viscosity of Water and its Binary Mixtures with Methanol and Ethanol by Molecular Simulation. J. Chem. Phys. 2011, 134, 074508.

(30) Guevara-Carrion, G.; Nieto-Draghi, C.; Vrabec, J.; Hasse, H. Prediction of Transport Properties by Molecular Simulation: Methanol and Ethanol and their Mixture. J. Phys. Chem. B 2008, 112, 16664-16674.

(31) Cussler, E. L. Mass Transfer in Fluid Systems, 2nd ed.; Cambridge University Press: Cambridge, 1997.

(32) Buzatu, D.; Buzatu, F. D.; Paduano, L.; Sartorio, R. Diffusion Coefficients for the Ternary System Water + Chloroform + Acetic Acid at 25 C. J. Solution Chem. 2007, 36, 1373-1384.

(33) Taylor, R.; Krishna, R. Multicomponent Mass Transfer; John Wiley \& Sons: New York, 1993.

(34) Krishna, R. Uphill diffusion in multicomponent mixtures. Chem. Soc. Rev. 2015, 44, 28122836.

(35) Green, M. S. Markoff Random Processes and the Statistical Mechanics of Time-Dependent Phenomena. II. Irreversible Processes in Fluids. J. Chem. Phys. 1954, 22, 398-414.

(36) Kubo, R. Statistical-Mechanical Theory of Irreversible Processes I. General Theory and Simple Applications to Magnetic and Conduction Problems. J. Phys. Soc. Jpn. 1957, 12, 570586.

(37) Meier, K.; Laesecke, A.; Kabelac, S. Transport Coefficients of the Lennard-Jones Model Fluid. II Self-Diffusion. J. Chem. Phys. 2004, 121, 9526-9535. 
(38) Sechenyh, V.; Legros, J. C.; Mialdun, A.; Ortiz de Zárate, J. M.; Shevtsova, V. Fickian Diffusion in Ternary Mixtures Composed by 1,2,3,4-Tetrahydronaphthalene, Isobutylbenzene, and n-Dodecane. J. Phys. Chem. B 2016, 120, 535-548.

(39) Abascal, J. L. F.; Vega, C. A General Purpose Model for the Condensed Phases of Water: TIP4P/2005. J. Chem. Phys. 2005, 123, 234505.

(40) Schnabel, T.; Vrabec, J.; Hasse, H. Henry's Law Constants of Methane, Nitrogen, Oxigen and Carbon dioxide in Ethanol from 273 to 498 K: Prediction from Molecular Simulation. Fluid Phase Equilib. 2005, 233, 134-143.

(41) Schnabel, T.; Srivastava, A.; Vrabec, J.; Hasse, H. Hydrogen Bonding of Methanol in Supercritical CO2: Comparison between 1H-NMR Spestroscopic Data and Molecular Simulation Results. J. Phys. Chem. B 2007, 111, 9871-9878.

(42) Allen, M. P.; Tildesley, D. J. Computer Simulation of Liquids; Clarendon Press: Oxford, 1987.

(43) Yeh, I. C.; Hummer, G. System-Size Dependence of Diffusion Coefficients and Viscosities from Molecular Dynamics Simulations with Periodic Boundary Conditions. J. Phys. Chem. B 2004, 108, 15873-15879.

(44) Heyes, D. M.; Cass, M. J.; Powles, J.; Evans, W. A. B. Self-Diffusion Coefficient of the HardSphere Fluid: System Size Dependence and Empirical Correlations. J. Phys. Chem. B 2007, $111,1455-1464$.

(45) Moultos, O. A.; Zhang, Y.; Tsimpanogiannis, I. N.; Economou, I. G.; Maginn, E. J. SystemSize Corrections for Self-Diffusion Coefficients Calculated from Molecular Dynamics Simulations: The Case of $\mathrm{CO}_{2}$, n-Alkanes, and Poly(ethylene Glycol) Dimethyl Ethers. J. Chem. Phys. 2016, 145, 074109. 
(46) Jamali, S. H.; Wolff, L.; Becker, T. M.; Bardow, A.; Vlugt, T. J. H.; Moultos, O. A. FiniteSize Effects of Binary Mutual Diffusion Coefficients from Molecular Dynamics. J. Chem. Theory Comput. 2018, 14, 2667-2677.

(47) Kirkwood, J. G.; Buff, F. P. The Statistical Mechanical Theory of Solutions. J. Chem. Phys. 1951, 774-777.

(48) Milzetti, J.; Nayar, D.; van der Vegt, N. F. A. Convergence of Kirkwood-Buff Integrals of Ideal and Nonideal Aqueous Solutions Using Molecular Dynamics Simulations. J. Phys. Chem. B 2018, 122, 5515-5526.

(49) Krüger, P.; Schnell, S. K.; Bedeaux, D.; Kjelstrup, S.; Vlugt, T. J. H.; Simon, J.-M. KirkwoodBuff Integrals for Finite Volumes. J. Phys. Chem. Lett. 2013, 4, 235-238.

(50) Ganguly, P.; van der Vegt, N. F. A. Convergence of Sampling Kirkwood-Buff Integrals of Aqueous Solutions with Molecular Dynamics Simulations. J. Chem. Theory Comput. 2013, 9, 1347-1355.

(51) Fingerhut, R.; Vrabec, J. Kirkwood-Buff integration: A Promising Route to Entropic Properties? Fluid Phase Equilib. 2019, 485, 270-281.

(52) Ben-Naim, A. Molecular Theory of Solutions; University Press: Oxford, 2006.

(53) Wilson, G. M. Vapor-Liquid Equilibrium. A New Expression for the Excess Free Energy of Mixing. J. Am. Chem. Soc. 1964, 86, 127-130. 


\title{
Supplementary Material to:
}

\section{Fick Diffusion Coefficient Matrix of a Quaternary Liquid Mixture by Molecular Dynamics}

\author{
Gabriela Guevara-Carrion, Robin Fingerhut, and Jadran Vrabec* \\ Thermodynamics and Process Engineering, Technical University Berlin, 10587 Berlin, \\ Germany \\ E-mail: vrabec@tu-berlin.de \\ Phone: +493031422755
}




\section{Ternary limits of quaternary Fick diffusion coefficients}

Here we present a complete analysis of the asymptotic behavior of diffusion coefficients for quaternary mixtures. The three independent diffusive fluxes in the molar-averaged frame of reference can be written as

$$
\begin{aligned}
& -J_{1} / \rho=D_{11}^{\text {quat }} \nabla x_{1}+D_{12}^{\text {quat }} \nabla x_{2}+D_{13}^{\text {quat }} \nabla x_{3}, \\
& -J_{2} / \rho=D_{21}^{\text {quat }} \nabla x_{1}+D_{22}^{\text {quat }} \nabla x_{2}+D_{23}^{\text {quat }} \nabla x_{3}, \\
& -J_{3} / \rho=D_{31}^{\text {quat }} \nabla x_{1}+D_{32}^{\text {quat }} \nabla x_{2}+D_{33}^{\text {quat }} \nabla x_{3} .
\end{aligned}
$$

When the water content of the studied quaternary mixture water $(1)+$ methanol $(2)+$ ethanol (3) + 2-propanol (4) vanishes, so does its as mass flux i.e., $\nabla x_{1} \rightarrow 0$ and $J_{1} \rightarrow 0$. Then, from Eq. (1) it follows that $D_{12}^{\text {quat }} \nabla x_{2}+D_{13}^{\text {quat }} \nabla x_{3} \rightarrow 0$. Since $\nabla x_{2}$ and $\nabla x_{3}$ are independent variables $D_{12}^{\text {quat }} \rightarrow 0$ and $D_{12}^{\text {quat }} \rightarrow 0$. Further, Eqs. (2) and (3) can be written as

$$
\begin{aligned}
& -J_{2} / \rho \rightarrow D_{22}^{\text {quat }} \nabla x_{2}+D_{23}^{\text {quat }} \nabla x_{3}, \\
& -J_{3} / \rho \rightarrow D_{32}^{\text {quat }} \nabla x_{2}+D_{33}^{\text {quat }} \nabla x_{3} .
\end{aligned}
$$

The two independent diffusive fluxes for the ternary mixture methanol $(2)+$ ethanol $(3)+$ 2-propanol (4) are

$$
\begin{aligned}
& -J_{2} / \rho=D_{11}^{\mathrm{tern}} \nabla x_{2}+D_{12}^{\mathrm{tern}} \nabla x_{3}, \\
& -J_{3} / \rho=D_{21}^{\mathrm{tern}} \nabla x_{2}+D_{22}^{\mathrm{tern}} \nabla x_{3} .
\end{aligned}
$$


From the comparison of Eq. (4) with Eq. (6) as well as Eq. (5) with Eq. (7), it follows that

$$
D_{22}^{\text {quat }} \rightarrow D_{11}^{\text {tern }}, \quad D_{23}^{\text {quat }} \rightarrow D_{12}^{\text {tern }}, \quad D_{32}^{\text {quat }} \rightarrow D_{21}^{\text {tern }}, \quad D_{33}^{\text {quat }} \rightarrow D_{22}^{\text {tern }}
$$

A similar analysis can be done for $x_{2} \rightarrow 0$ and $x_{3} \rightarrow 0$. However, the asymptotic behavior for $x_{4} \rightarrow 0$ requires a slightly different approach. If $x_{4} \rightarrow 0$, it follows from the condition $x_{1}+x_{2}+x_{3}+x_{4}=1$ that $\nabla x_{3} \rightarrow-\nabla x_{1}-\nabla x_{2}$ and the expressions for the diffusive fluxes $J_{1}^{V}$ and $J_{2}^{V}$ take the form

$$
\begin{aligned}
& -J_{1} / \rho \rightarrow\left(D_{11}^{\text {quat }}-D_{13}^{\text {quat }}\right) \nabla x_{1}-\left(D_{11}^{\text {quat }}-D_{13}^{\text {quat }}\right) \nabla x_{2}, \\
& -J_{2} / \rho \rightarrow\left(D_{21}^{\text {quat }}-D_{23}^{\text {quat }}\right) \nabla x_{1}-\left(D_{22}^{\text {quat }}-D_{23}^{\text {quat }}\right) \nabla x_{2} .
\end{aligned}
$$

Taking into account the two independent diffusive fluxes for the ternary mixture water (1) + methanol (2) + ethanol (3) leads to

$$
\begin{aligned}
& -J_{1} / \rho=D_{11}^{\mathrm{tern}} \nabla x_{1}+D_{12}^{\mathrm{tern}} \nabla x_{2}, \\
& -J_{1} / \rho=D_{21}^{\mathrm{tern}} \nabla x_{1}+D_{22}^{\mathrm{tern}} \nabla x_{2} .
\end{aligned}
$$

Applying the same logic as above, it follows that

$$
\begin{array}{ll}
D_{11}^{\text {quat }}-D_{13}^{\text {quat }} \rightarrow D_{11}^{\text {tern }}, & D_{12}^{\text {quat }}-D_{13}^{\text {quat }} \rightarrow D_{12}^{\text {tern }}, \\
D_{21}^{\text {quat }}-D_{23}^{\text {quat }} \rightarrow D_{21}^{\text {tern }}, & D_{22}^{\text {quat }}-D_{23}^{\text {quat }} \rightarrow D_{22}^{\text {tern }} .
\end{array}
$$




\section{Simulation details}

Molecular dynamics simulations were performed with the program $m s 2^{1}$ in two steps: First, a simulation in the isobaric-isothermal $(N p T)$ ensemble was carried out to calculate the density at the desired temperature, pressure and composition. In the second step, a canonic $(N V T)$ ensemble simulation was performed at the corresponding thermodynamic conditions to simultaneously determine the phenomenological coefficient and thermodynamic factor matrices. Newton's equations of motion were solved with a fifth-order Gear predictor-corrector numerical integrator and the temperature was controlled by velocity scaling. Throughout, the integration time step was 0.93 fs. The simulations contained 6000 molecules and were carried out in a cubic volume with periodic boundary conditions, where the cut-off radius was set to $r_{c}=24.5 \AA$. Lennard-Jones long range interactions were considered using angle averaging. ${ }^{2}$ Electrostatic long-range corrections were approximated by the reaction field technique with conducting boundary conditions $\left(\epsilon_{R F}=\infty\right)$.

The simulations in the $N p T$ ensemble were equilibrated over $4 \times 10^{5}$ time steps, followed by a production run over $3 \times 10^{6}$ time steps. In the $N V T$ ensemble, the simulations were equilibrated over $5 \times 10^{5}$ time steps, followed by production runs of $1.4 \times 10^{8}$ time steps. The phenomenological coefficients were calculated for up to $1.1 \times 10^{6}$ independent time origins of the autocorrelation functions. The sampling length of the autocorrelation functions was 17.5 ps throughout. The separation between the time origins was chosen such that all autocorrelation functions have decayed at least to 1 /e of their normalized value to achieve

their time independence. ${ }^{3}$ The uncertainties of the predicted values were estimated with a block averaging method. ${ }^{4}$ 


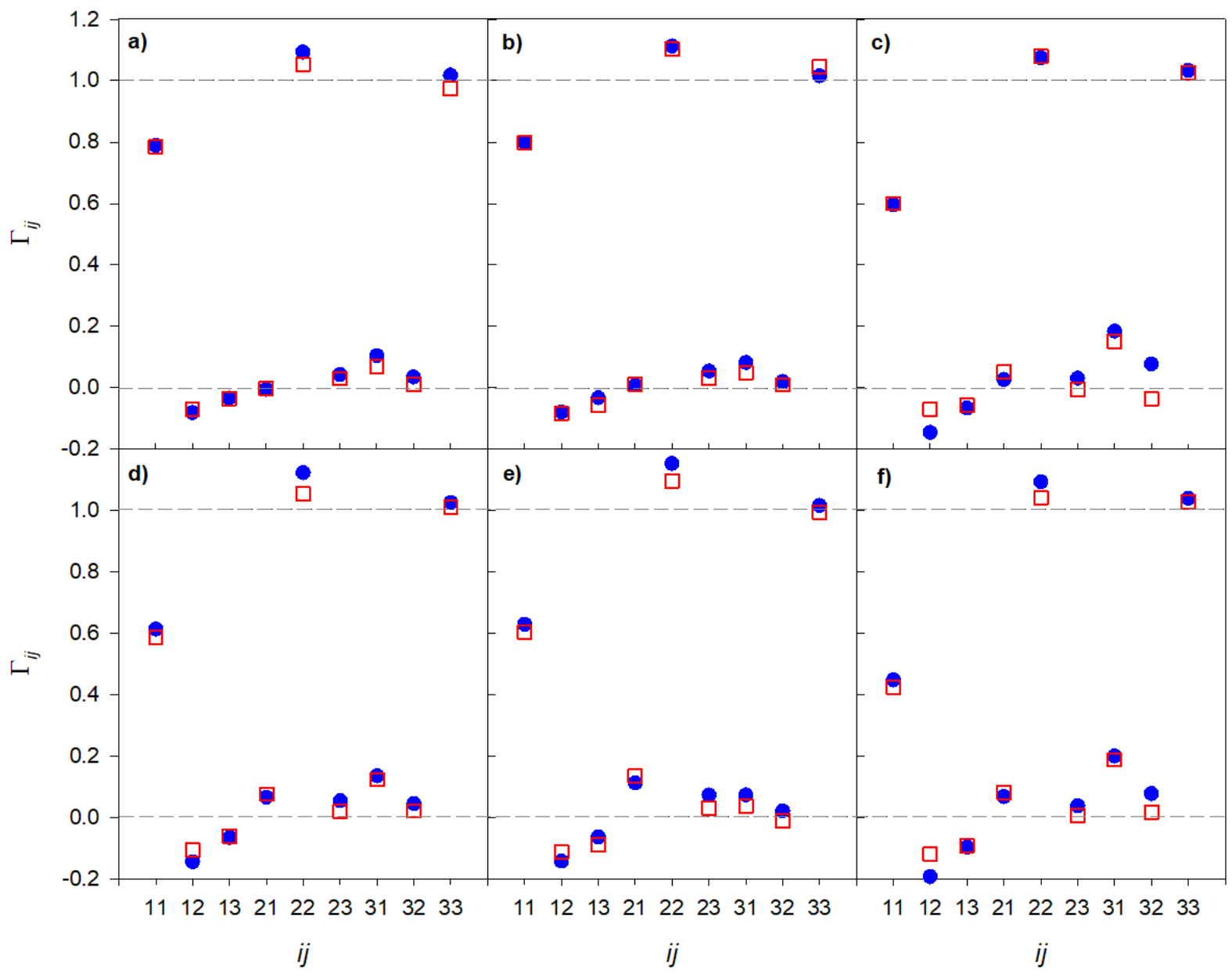

Figure S1: Elements of the thermodynamic factor matrix $\Gamma_{i j}$ for six quaternary compositions; a) $x_{1}=0.125 \mathrm{~mol} \mathrm{~mol}^{-1}, x_{2}=0.25 \mathrm{~mol} \mathrm{~mol}^{-1}$ and $x_{3}=0.375 \mathrm{~mol} \mathrm{~mol}^{-1}$; b) $x_{1}=0.125$ mol mol${ }^{-1}, x_{2}=0.375 \mathrm{~mol} \mathrm{~mol}^{-1}$ and $x_{3}=0.25 \mathrm{~mol} \mathrm{~mol}^{-1}$; c) $x_{1}=0.25 \mathrm{~mol} \mathrm{~mol}^{-1}, x_{2}=$ $0.125 \mathrm{~mol} \mathrm{~mol}^{-1}$ and $x_{3}=0.375 \mathrm{~mol} \mathrm{~mol}{ }^{-1}$; d) $x_{1}=0.25 \mathrm{~mol} \mathrm{~mol}^{-1}, x_{2}=0.25 \mathrm{~mol} \mathrm{~mol}^{-1}$ and $x_{3}=0.25 \mathrm{~mol} \mathrm{~mol}^{-1}$; e) $x_{1}=0.25 \mathrm{~mol} \mathrm{~mol}^{-1}, x_{2}=0.375 \mathrm{~mol} \mathrm{~mol}^{-1}$ and $x_{3}=0.125 \mathrm{~mol}$ $\mathrm{mol}^{-1}$; f) $x_{1}=0.375 \mathrm{~mol} \mathrm{~mol}^{-1}, x_{2}=0.125 \mathrm{~mol} \mathrm{~mol}^{-1}$ and $x_{3}=0.25 \mathrm{~mol} \mathrm{~mol}^{-1}$. Present quaternary results based on Kirkwood-Buff integration (red symbols) are compared with $\Gamma_{i j}$ calculated with the Wilson excess Gibbs energy model fitted to binary simulation results (blue symbols). The statistical uncertainties are within symbol size. 

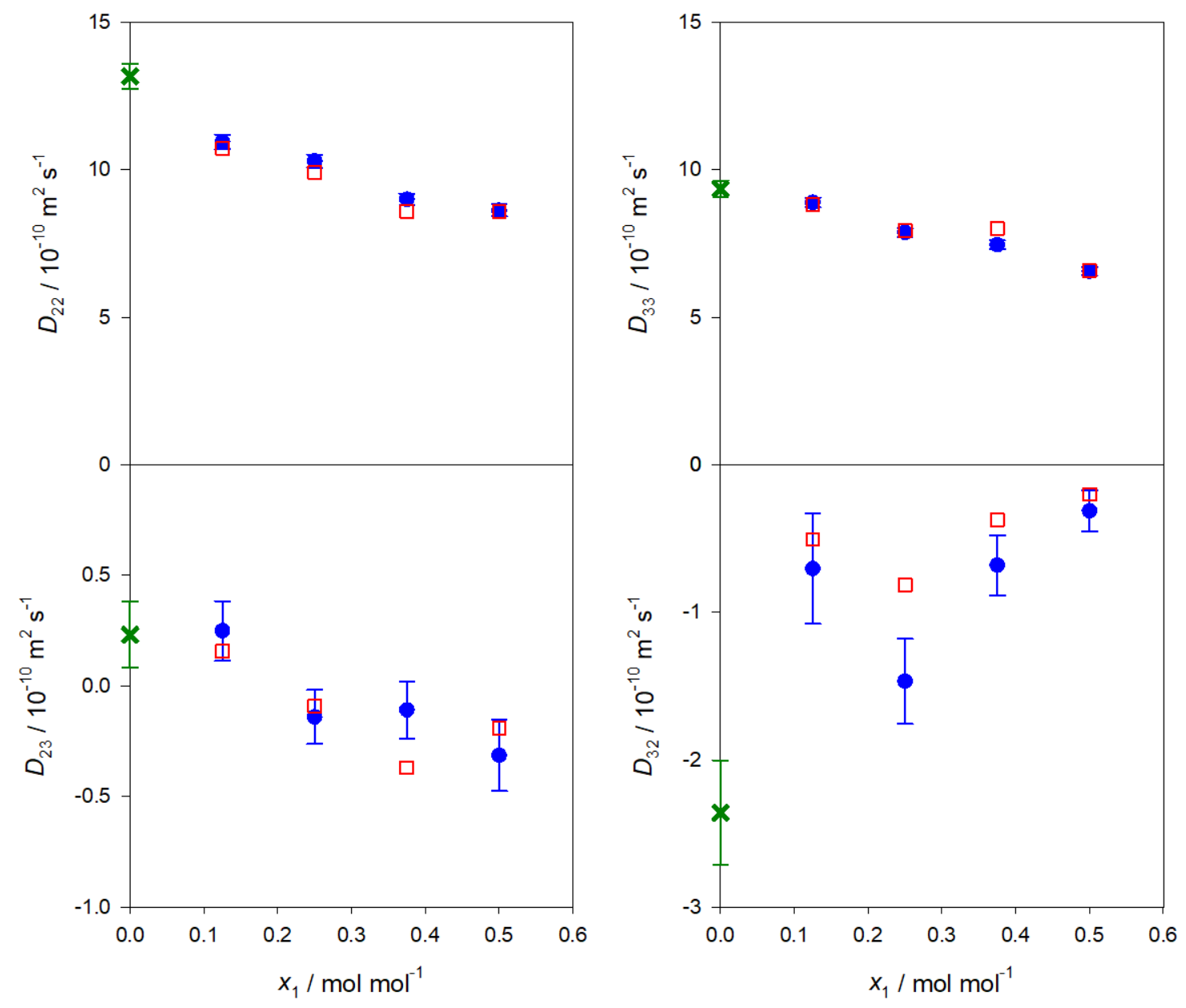

Figure S2: Main and cross elements of the Fick diffusion coefficient matrix as a function of water mole fraction for selected compositions with $x_{2}=0.125 \mathrm{~mol} \mathrm{~mol}^{-1}$ and $x_{4}=0.25 \mathrm{~mol}$ $\mathrm{mol}^{-1}$. Simulation results for the quaternary mixture (blue symbols) are shown together with the results of the diffusion matrix of the ternary subsystem where $x_{1} \rightarrow 0$ (green symbol) and the predictive equations by Allie-Ebrahim et al. ${ }^{5}$ (red symbols). 

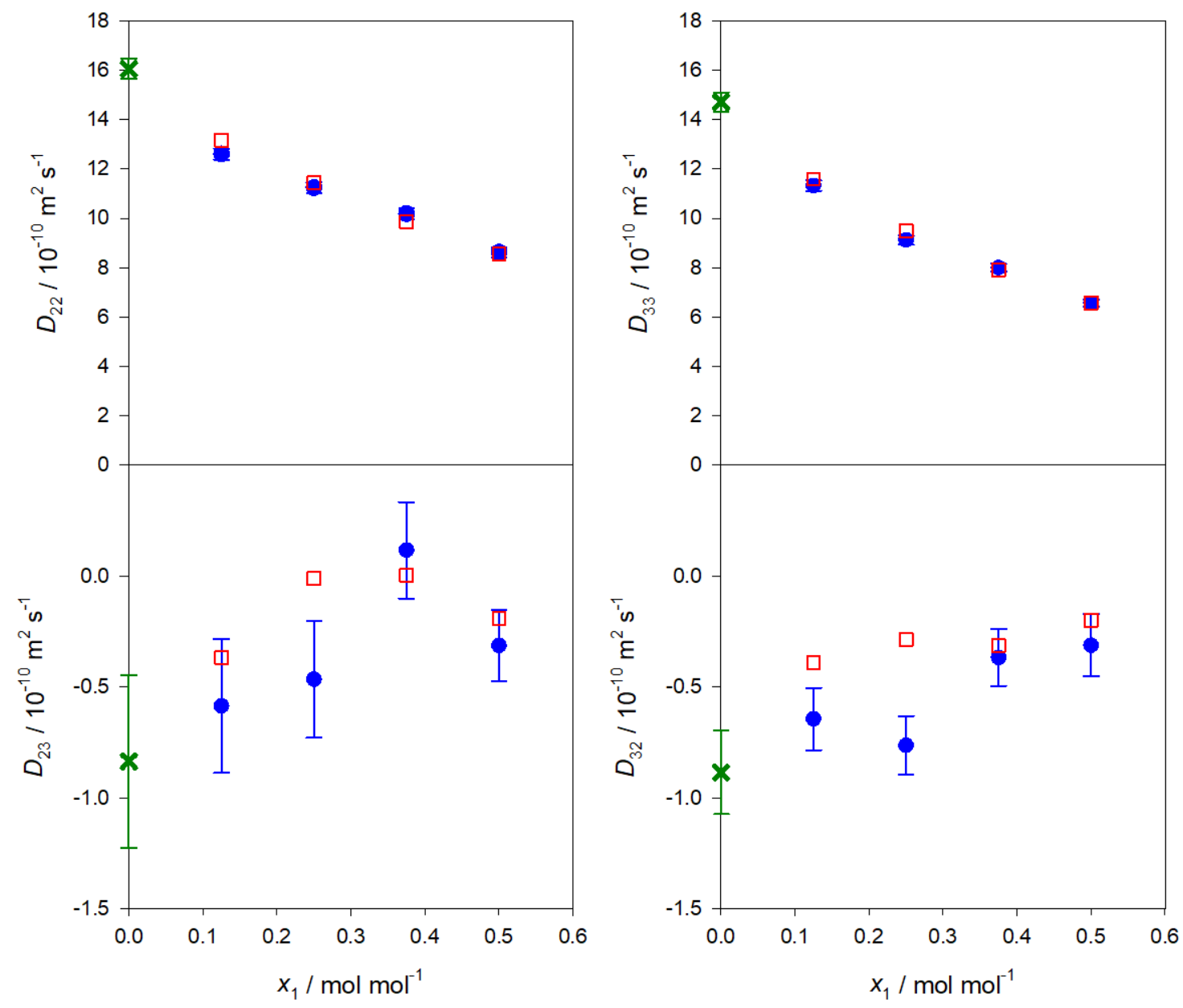

Figure S3: Main and cross elements of the Fick diffusion coefficient matrix as a function of water mole fraction for selected compositions with $x_{3}=0.125 \mathrm{~mol} \mathrm{~mol}^{-1}$ and $x_{4}=0.25 \mathrm{~mol}$ $\mathrm{mol}^{-1}$. Simulation results for the quaternary mixture (blue symbols) are shown together with the results of the diffusion matrix of the ternary subsystem where $x_{1} \rightarrow 0$ (green symbol) and the predictive equations by Allie-Ebrahim et al. ${ }^{5}$ (red symbols). 

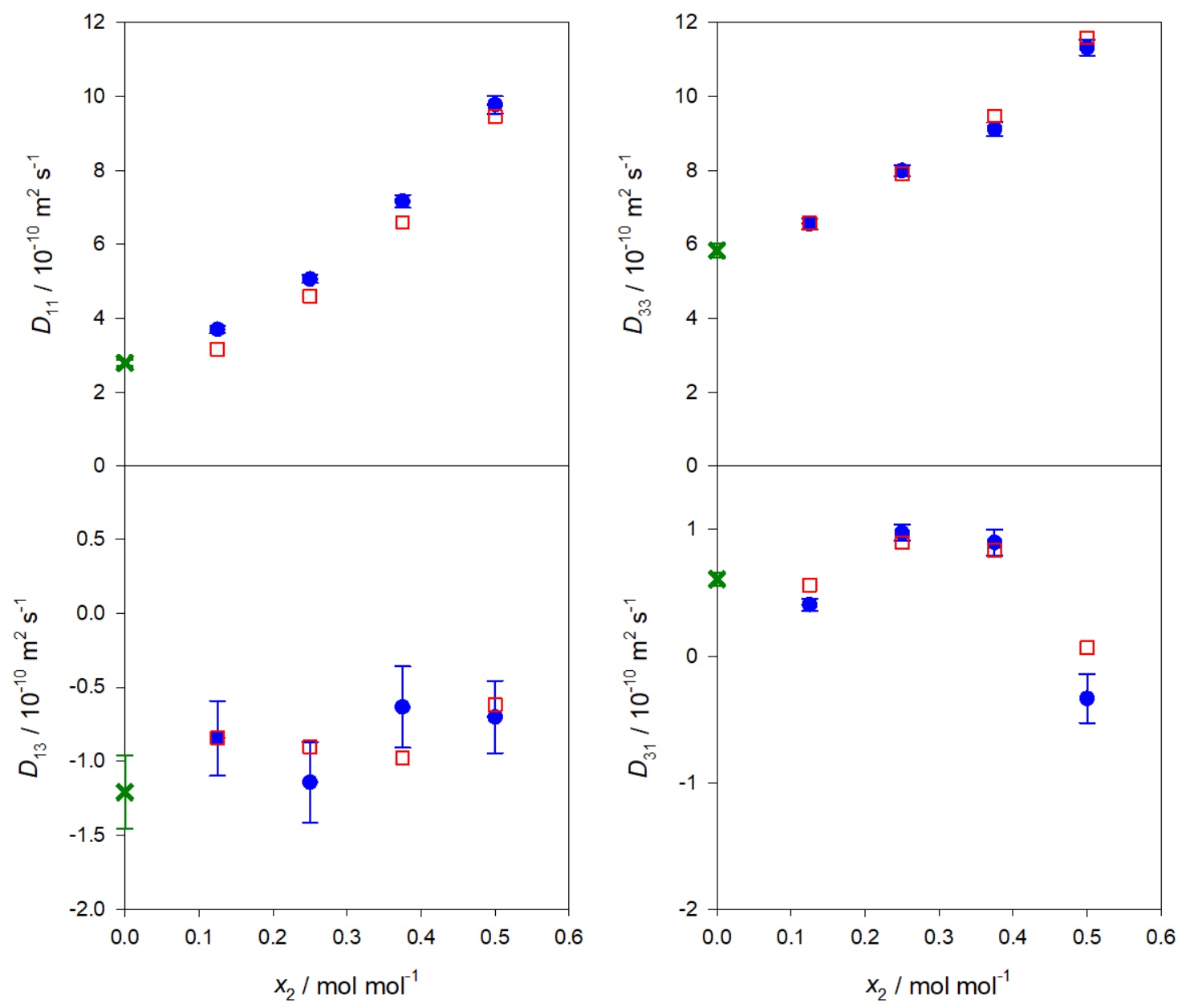

Figure S4: Main and cross elements of the Fick diffusion coefficient matrix as a function of methanol mole fraction for selected compositions with $x_{3}=0.125 \mathrm{~mol} \mathrm{~mol}^{-1}$ and $x_{4}=$ $0.25 \mathrm{~mol} \mathrm{~mol}^{-1}$. Simulation results for the quaternary mixture (blue symbols) are shown together with the results of the diffusion matrix of the ternary subsystem where $x_{2} \rightarrow 0$ (green symbol) and the predictive equations by Allie-Ebrahim et al. ${ }^{5}$ (red symbols). 

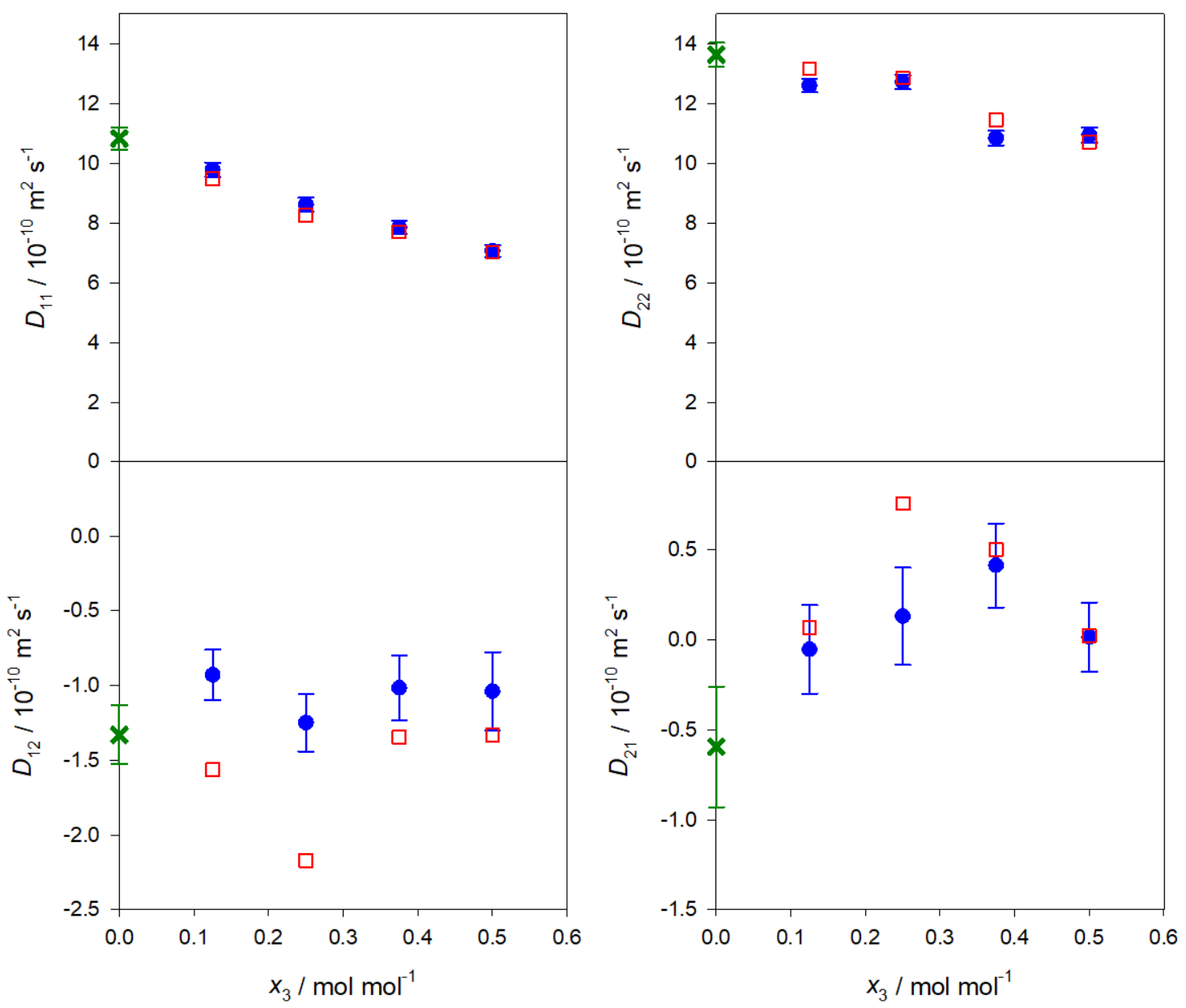

Figure S5: Main and cross elements of the Fick diffusion coefficient matrix as a function of ethanol mole fraction for selected compositions with $x_{1}=0.125 \mathrm{~mol} \mathrm{~mol}^{-1}$ and $x_{4}=$ $0.25 \mathrm{~mol} \mathrm{~mol}^{-1}$. Simulation results for the quaternary mixture (blue symbols) are shown together with the results of the diffusion matrix of the ternary subsystem where $x_{3} \rightarrow 0$ (green symbol) and the predictive equations by Allie-Ebrahim et al. ${ }^{5}$ (red symbols). 

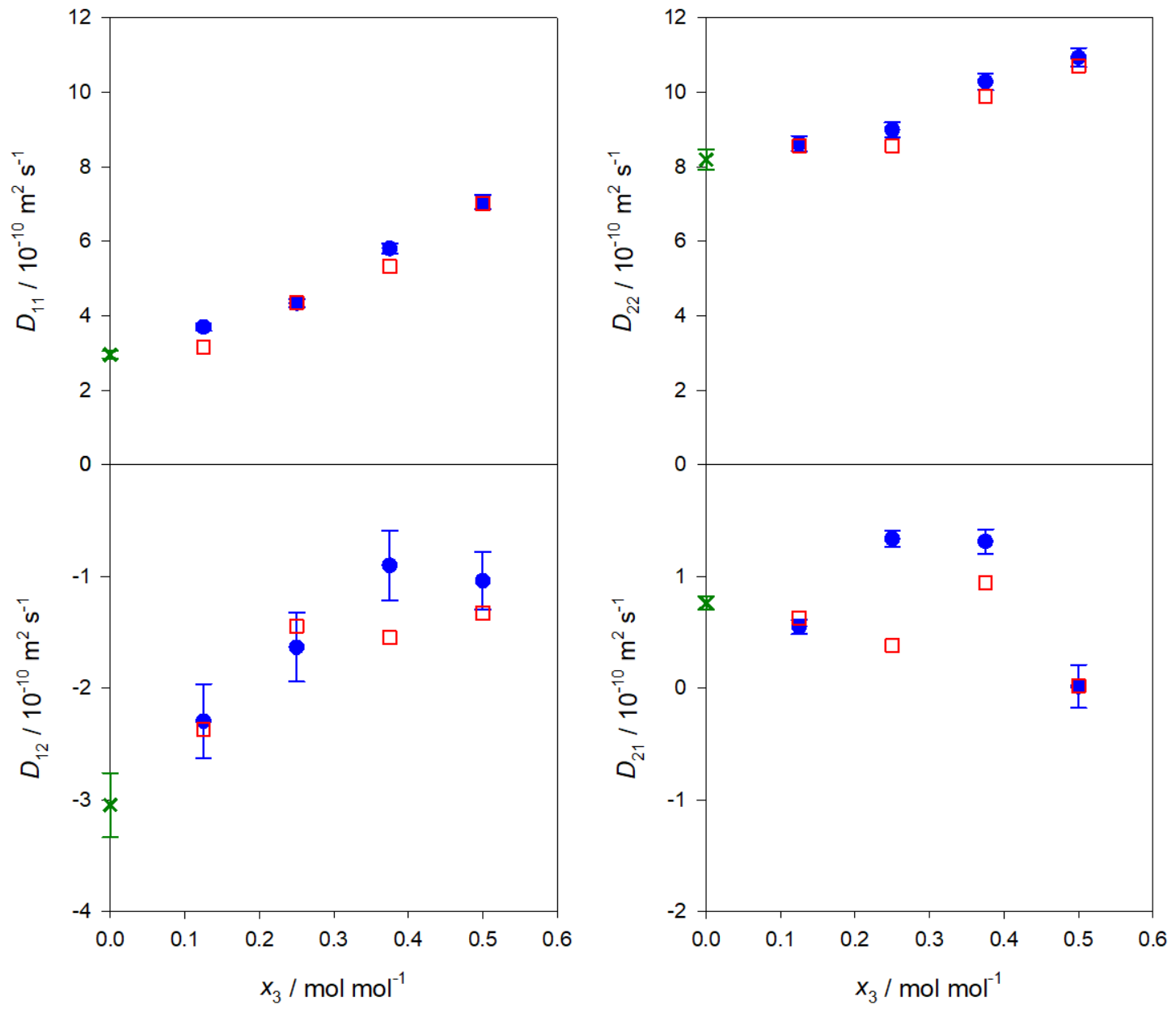

Figure S6: Main and cross elements of the Fick diffusion coefficient matrix as a function of ethanol mole fraction for selected compositions with $x_{2}=0.125 \mathrm{~mol} \mathrm{~mol}^{-1}$ and $x_{4}=$ $0.25 \mathrm{~mol} \mathrm{~mol}^{-1}$. Simulation results for the quaternary mixture (blue symbols) are shown together with the results of the diffusion matrix of the ternary subsystem where $x_{3} \rightarrow 0$ (green symbol) and the predictive equations by Allie-Ebrahim et al. ${ }^{5}$ (red symbols). 


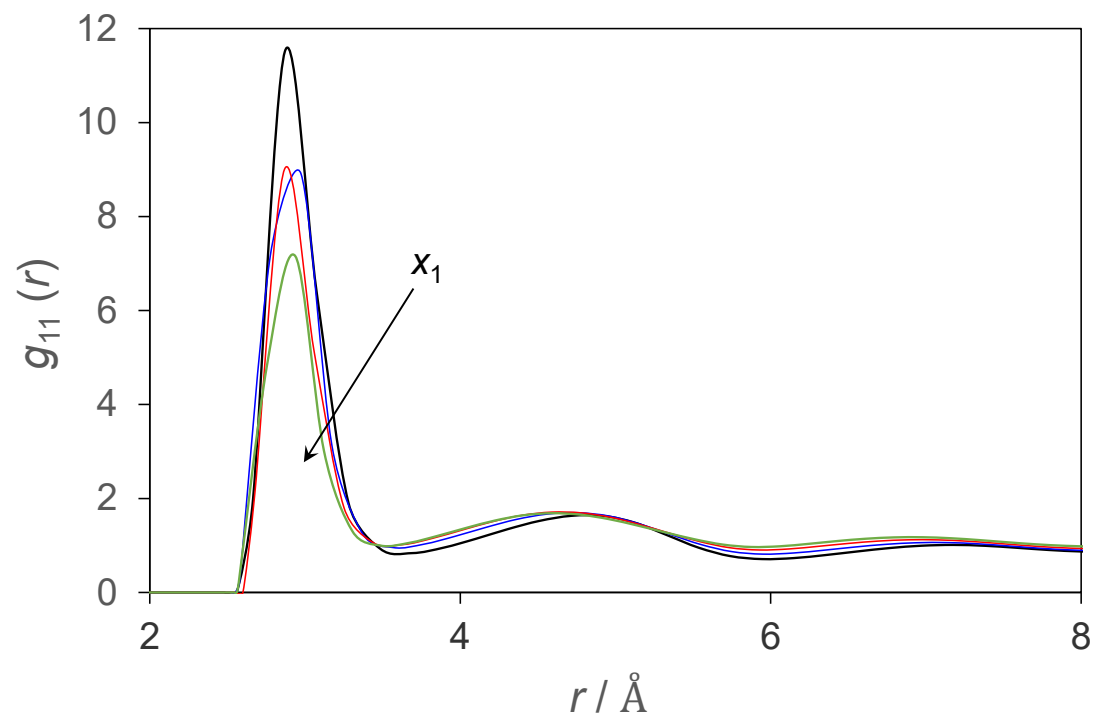

Figure S7: Water-water radial distribution function $g_{11}(r)$ for varying water mole fraction $x_{1}$ $=0.125 \mathrm{~mol} \mathrm{~mol}^{-1}$ (black line), $0.25 \mathrm{~mol} \mathrm{~mol}^{-1}$ (blue line), $0.375 \mathrm{~mol} \mathrm{~mol}^{-1}$ (red line) and $0.5 \mathrm{~mol} \mathrm{~mol}^{-1}$ (green line) with constant $x_{2}=0.125 \mathrm{~mol} \mathrm{~mol}^{-1}$ and $x_{4}=0.25 \mathrm{~mol} \mathrm{~mol}^{-1}$. 


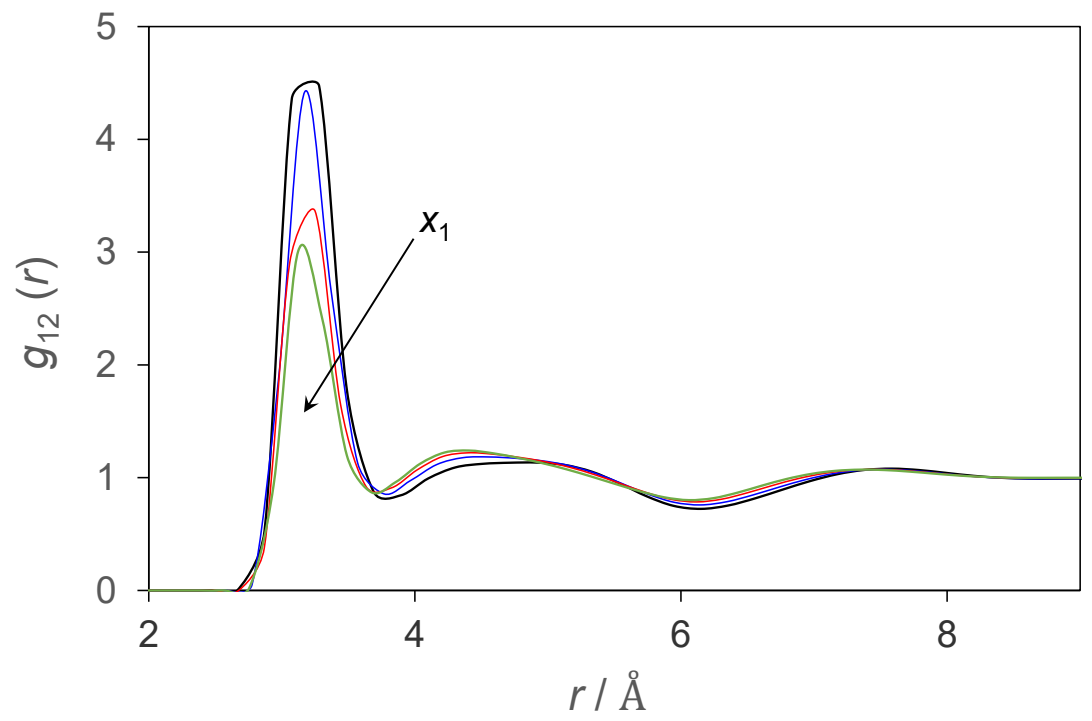

Figure S8: Water-methanol radial distribution function $g_{12}(r)$ for varying water mole fraction $x_{1}=0.125 \mathrm{~mol} \mathrm{~mol}^{-1}$ (black line), $0.25 \mathrm{~mol} \mathrm{~mol}^{-1}$ (blue line), $0.375 \mathrm{~mol} \mathrm{~mol}^{-1}$ (red line) and $0.5 \mathrm{~mol} \mathrm{~mol}^{-1}$ (green line) with constant $x_{2}=0.125 \mathrm{~mol} \mathrm{~mol}^{-1}$ and $x_{4}=0.25 \mathrm{~mol} \mathrm{~mol}^{-1}$. 


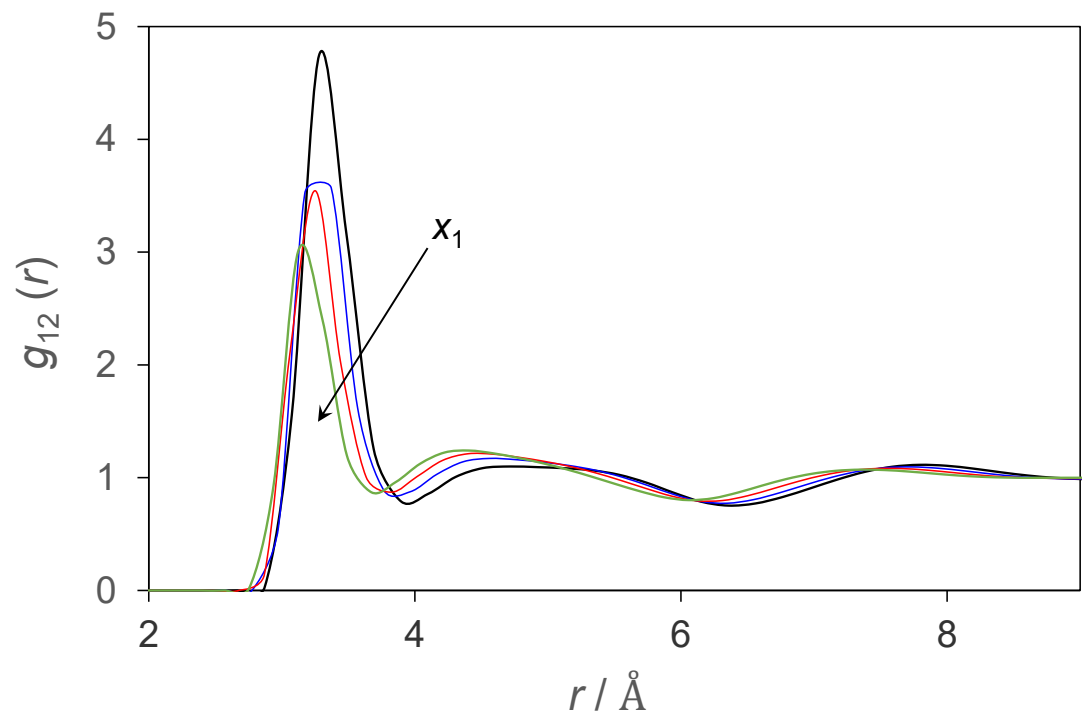

Figure S9: Water-methanol radial distribution function $g_{12}(r)$ for varying water mole fraction $x_{1}=0.125 \mathrm{~mol} \mathrm{~mol}^{-1}$ (black line), $0.25 \mathrm{~mol} \mathrm{~mol}^{-1}$ (blue line), $0.375 \mathrm{~mol} \mathrm{~mol}^{-1}$ (red line) and $0.5 \mathrm{~mol} \mathrm{~mol}^{-1}$ (green line) with constant $x_{3}=0.125 \mathrm{~mol} \mathrm{~mol}^{-1}$ and $x_{4}=0.25 \mathrm{~mol} \mathrm{~mol}^{-1}$. 


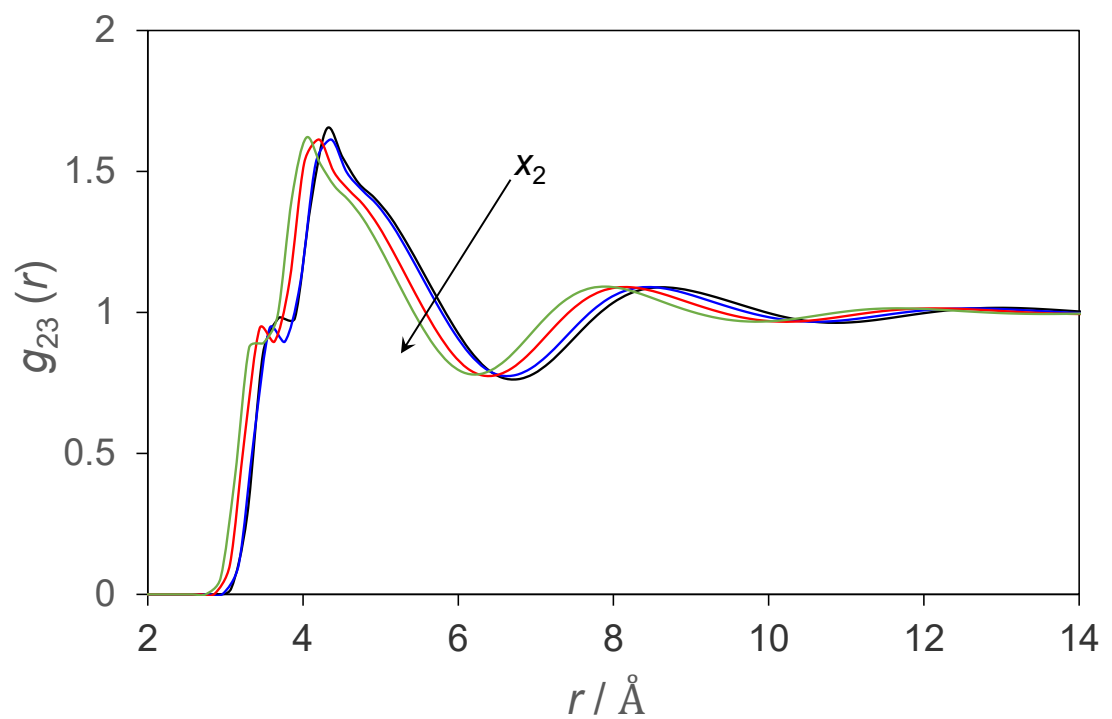

Figure S10: Methanol-ethanol radial distribution function $g_{23}(r)$ for varying methanol mole fraction $x_{2}=0.125 \mathrm{~mol} \mathrm{~mol}^{-1}$ (black line), $0.25 \mathrm{~mol} \mathrm{~mol}^{-1}$ (blue line), $0.375 \mathrm{~mol} \mathrm{~mol}^{-1}$ (red line) and $0.5 \mathrm{~mol} \mathrm{~mol}^{-1}$ (green line) with constant $x_{1}=0.125 \mathrm{~mol} \mathrm{~mol}^{-1}$ and $x_{4}=0.25 \mathrm{~mol}$ $\mathrm{mol}^{-1}$. 


\section{References}

(1) Glass, C. W.; Reiser, S.; Rutkai, G.; Deublein, S.; Köster, A.; Guevara-Carrion, G.; Wafai, A.; Horsch, M.; Bernreuther, M.; Windmann, T. et al. ms2: A Molecular Simulation Tool for Thermodynamic Properties, New Version Release. Comp. Phys. Commun. 2014, 185, 3302-3306.

(2) Lustig, R. Angle-average for the powers of the distance between two separated vectors. Mol. Phys. 1988, 65, 175-179.

(3) Schoen, M.; Hoheisel, C. The mutual diffusion coefficient D_12 in binary liquid model mixtures. Molecular dynamics calculations based on Lennard-Jones (12-6) potentials. Mol. Phys. 1984, 52, 33-56.

(4) Allen, M. P.; Tildesley, D. J. Computer Simulation of Liquids; Clarendon Press: Oxford, 1987.

(5) Allie-Ebrahim, T.; Russo, V.; Ortona, O.; Paduano, L.; Tesser, R.; Di Serio, M.; Singh, P.; Zhu, Q.; Moggridge, G. D.; D’Agostino, C. A Predictive Model for the Diffusion of a Highly Non-Ideal Ternary System. Phys. Chem. Chem. Phys. 2018, 20, $18436-18446$. 